\title{
TAMENESS OF JOINS INVOLVING THE PSEUDOVARIETY OF LOCAL SEMILATTICES
}

\author{
J. C. COSTA \\ Centro de Matemática e Departamento de Matemática e Aplicações \\ Universidade do Minho, Campus de Gualtar, 4700-320 Braga, Portugal \\ Email: jcosta@math.uminho.pt \\ C. NOGUEIRA \\ Escola Superior de Tecnologia e Gestão, Instituto Politécnico de Leiria \\ Campus 2, Morro do Lena, Alto Vieiro, 2411-901 Leiria, Portugal \\ Email: conceicao.veloso@ipleiria.pt
}

July 2, 2012

\begin{abstract}
In this paper we prove that, if $\mathbf{V}$ is a $\kappa$-tame pseudovariety which satisfies the pseudoidentity $x y^{\omega+1} z=x y z$, then the pseudovariety join $\mathbf{L S I} \vee \mathbf{V}$ is also $\kappa$-tame. Here, $\mathbf{L S l}$ denotes the pseudovariety of local semilattices and $\kappa$ denotes the implicit signature consisting of the multiplication and the $(\omega-1)$-power. As a consequence, we deduce that $\mathbf{L S I} \vee \mathbf{V}$ is decidable. In particular the joins $\mathbf{L S} \mathbf{\vee} \mathbf{A b}, \mathbf{L S} \mathbf{\vee} \mathbf{G}, \mathbf{L S l} \vee \mathbf{O C R}$ and $\mathbf{L S l} \vee \mathbf{C R}$ are decidable.
\end{abstract}

Keywords: Semigroup, local semillatice, tame pseudovariety, join of pseudovarieties, pseudoword, graph equation system

Mathematics Subject Classification 2000: 20M05, 20M07, 20M35

\section{Introduction}

A pseudovariety of semigroups is a class of finite semigroups closed under taking subsemigroups, homomorphic images and finite direct product. It is said to be decidable if its membership problem has a solution, that is, if there is an algorithm to test whether a given finite semigroup lies in that pseudovariety. The join $\mathbf{V} \vee \mathbf{W}$ of two pseudovarieties $\mathbf{V}$ and $\mathbf{W}$ is the least pseudovariety containing both $\mathbf{V}$ and $\mathbf{W}$. A well-known result of Albert, Baldinger and Rhodes [1] states that the join of two decidable pseudovarieties may not be decidable. Decidability also fails to be preserved by some other common pseudovariety operators, such as semidirect product, block product, Mal'cev product and power [22, 12].

An idea which has been recently explored by several authors is to impose stronger properties on the pseudovarieties upon which the operators are to be applied that guarantee that the resulting pseudovarieties are decidable [3, 23]. When it was introduced, by Almeida and Steinberg [4], the notion of tameness seamed to be the suitable property for the case of the semidirect product operator, but that assertion is still not proved. However, many pseudovarieties obtained from tame pseudovarieties using for instance the join operator are expected 
to be decidable, although this is in general apparently not trivial to prove. The pseudovariety LSl [17] is known to be tame. We show in this paper that the joins of LSl with certain pseudovarieties are not only decidable but also tame themselves.

The tameness property is parameterized by an implicit signature $\sigma$, and we speak of $\sigma$ tameness. Proving the $\sigma$-tameness of a pseudovariety $\mathbf{V}$ involves proving two properties: that the word problem for $\sigma$-terms over $\mathbf{V}$ is decidable, and that $\mathbf{V}$ is $\sigma$-reducible, which means, informally speaking, the following: given a finite graph equation system with rational constraints, the existence of a solution in the free profinite semigroup over $\mathbf{V}$ implies the existence of a solution given by $\sigma$-terms of the system over $\mathbf{V}$ satisfying the same constraints.

The canonical signature $\kappa$, containing the semigroup multiplication and the $(\omega-1)$-power, is the most commonly used signature. For instance, a non-exhaustive list of pseudovarieties already known to be $\kappa$-tame is given by: $\mathbf{K}$ of semigroups in which idempotents are left zeros [10], LSl [17], $\mathbf{J}$ of $\mathcal{J}$-trivial semigroups [3], $\mathbf{R}$ of $\mathcal{R}$-trivial semigroups [5], Ab of abelian groups [7], $\mathbf{G}$ of groups [11, 4], OCR of orthodox completely regular semigroups [8], and $\mathbf{C R}$ of completely regular semigroups [9] (a property on which this result depends was later observed by K. Auinger). We notice that LSl [16], R [6] and Ab [7] enjoy a stronger property, named complete $\kappa$-tameness, where general finite systems of equations of $\sigma$-terms are considered, while $\mathbf{G}$ is not completely $\kappa$-tame [18].

We establish $\kappa$-tameness of joins of the form $\mathbf{L S I} \vee \mathbf{V}$, where $\mathbf{V}$ is any $\kappa$-tame pseudovariety which satisfies the pseudoidentity $x y^{\omega+1} z=x y z$. In particular, it follows that the pseudovarieties $\mathbf{L S l} \vee \mathbf{A b}, \mathbf{L S l} \vee \mathbf{G}, \mathbf{L S l} \vee \mathbf{O C R}$ and $\mathbf{L S l} \vee \mathbf{C R}$ are $\kappa$-tame and, so, decidable. There are several examples of $\kappa$-tame pseudovarieties of the form $\mathbf{W} \vee \mathbf{V}$ in the literature. This is the case, for instance, of $\mathbf{K} \vee \mathbf{V}$ [15] and $\mathbf{J} \vee \mathbf{V}$ [5] for any $\kappa$-tame pseudovariety $\mathbf{V}(\mathbf{J} \vee \mathbf{V}$ was previously known to be $\kappa$-tame for $\mathbf{V} \subseteq \mathbf{C R}$ [24]), and $\mathbf{R} \vee \mathbf{V}$ [5] for any $\kappa$-tame pseudovariety which satisfies the pseudoidentity $x_{1} \cdots x_{r} y^{\omega+1} z t^{\omega}=x_{1} \cdots x_{r} y z t^{\omega}$.

This work is organized as follows. In a section of preliminaries, we recall the usual terminology and notation about words, pseudovarieties, graphs and $\sigma$-reducibility and review some basic results. Section 3 is devoted to recall the basic facts about the pseudovariety LSI, namely the solution of the $\kappa$-word problem for LSI. We next present some technical results on combinatorics of words which are essential for our purpose. Finally, we prove the $\kappa$-reducibility of some pseudovariety joins of the form $\mathbf{L S I} \vee \mathbf{V}$ in sections 5 and 6 .

\section{Preliminaries}

This section presents a brief description of the basic definitions, notation and results that are relevant to our study. The reader is referred to $[2,3]$ for general background about the classical theories of semigroups, pseudovarieties and profinite semigroups, and to [19] for further details about combinatorics on words.

\subsection{Words}

For a finite non-empty set, called an alphabet, we denote by $A^{+}$(resp. $\left.A^{*}\right)$ the free semigroup (resp. the free monoid) generated by $A$. An element $w$ of $A^{*}$ is called a (finite) word and its length is denoted by $|w|$. A word is said to be primitive if it cannot be written in the form $u^{n}$ 
with $n>1$. We say that two words $w$ and $z$ are conjugate if there exist words $u, v \in A^{*}$ such that $w=u v$ and $z=v u$. Note that, if $w$ is a primitive word and $z$ is a conjugate of $w$, then $z$ is also primitive. Let an order be fixed for the letters of the alphabet A. A Lyndon word is a primitive word which is minimal, with respect to the lexicographic ordering, in its conjugacy class.

A bi-infinite (resp. right-infinite, left-infinite) word on $A$ is a sequence $w=\left(a_{n}\right)_{n}$ of letters of $A$ indexed by $\mathbb{Z}$ (resp. $\mathbb{N},-\mathbb{N}$ ), also written

$$
w=\cdots a_{-2} a_{-1} \cdot a_{0} a_{1} a_{2} \cdots \quad\left(\text { resp. } w=a_{1} a_{2} a_{3} \cdots, w=\cdots a_{-3} a_{-2} a_{-1}\right) .
$$

We denote $w(n)=a_{n}$ and say that $a_{n}$ is the letter of $w$ at position $n$. The sets of bi-infinite, right-infinite and left-infinite words on $A$ will be denoted, respectively, by $A^{\mathbb{Z}}, A^{\mathbb{N}}$ and $A^{-\mathbb{N}}$.

We let $A^{\infty}=A^{+} \cup A^{\mathbb{N}}$ and $A^{-\infty}=A^{+} \cup A^{-\mathbb{N}}$. The product of two elements $w, z \in A^{\infty}$ is defined as follows: if $w, z \in A^{+}$, then $w z$ is defined as usual; right-infinite words are left zeros; finally, if $w=a_{1} \cdots a_{n}\left(a_{i} \in A\right)$ is a finite word and $z=b_{1} b_{2} \cdots\left(b_{j} \in A\right)$ is a right-infinite word, then $w z$ is the right-infinite word $w z=a_{1} \cdots a_{n} b_{1} b_{2} \cdots$. The product of elements of $A^{-\infty}$ is defined symmetrically. Notice that, endowed with these products, $A^{\infty}$ and $A^{-\infty}$ are semigroups. A word $x \in A^{*}$ is a prefix of a word $w \in A^{\infty}$, and $w$ is a right-extension of $x$, if there exists $z \in A^{\infty}$ such that $w=x z$. Dually, $x \in A^{*}$ is a suffix of $w \in A^{-\infty}$, and $w$ is a left-extension of $x$, if there exists $z \in A^{-\infty}$ such that $w=z x$. We denote by $p_{k}(w)$ (resp. $\left.s_{k}(w)\right)$ the prefix (resp. suffix) of length $k$ of $w \in A^{\infty}$ (resp. $w \in A^{-\infty}$ ).

Let $w$ be a (finite or infinite) word. For integers $i$ and $j$ such that $i \leq j$, we denote by $w[i, j]$ the word $a_{i} \cdots a_{j}$. A word $x \in A^{*}$ is a factor of $w$, and $w$ is an extension of $x$, if $x$ is the empty-word or $x$ is of the form $w[i, j]$. In such case, $w[i, j]$ is said to be an occurrence of the factor $x$ in $w$. We say that two occurrences $w[i, j]$ and $w[k, \ell]$ of factors in a word $w$ are disjoint (or that they do not overlap) if the integer intervals $[i, j]$ and $[k, \ell]$ are disjoint sets. For each pair of words $w, x \in A^{*}$, we denote by $\operatorname{occ}(x, w)$ the number of occurrences of $x$ in $w$, and by $\operatorname{docc}(x, w)$ the maximum number of disjoint occurrences of $x$ in $w$.

A right-infinite word of the form $v u^{+\infty}=v u u u \cdots$, with $u \in A^{+}$and $v \in A^{*}$, is said to be ultimately periodic and $u$ is said to be a period of $v u^{+\infty}$. Ultimately periodic left-infinite words are defined symmetrically as being words of the form $u^{-\infty} v=\cdots u u u v$. An ultimately periodic word $w \in A^{\mathbb{N}}$ (resp. $w \in A^{-\mathbb{N}}$ ) which can be written in the form $w=u^{+\infty}$ (resp. $w=u^{-\infty}$ ) for some $u \in A^{+}$, is said to be periodic.

A bi-infinite word $w$ is said to be left-ultimately periodic (resp. right-ultimately periodic) if $w=x \cdot y$ for some ultimately periodic left-infinite word $x \in A^{-\mathbb{N}}$ (resp. right-infinite word $\left.y \in A^{\mathbb{N}}\right)$. The word $w$ is said to be ultimately periodic if it is both left-ultimately and rightultimately periodic, and it is said to be periodic if one can choose $x=u^{-\infty}$ and $y=u^{+\infty}$ for some $u \in A^{+}$.

We define the shift operator $\sigma$ on $A^{\mathbb{Z}}$ by setting, for each $w=\left(a_{i}\right)_{i \in \mathbb{Z}} \in A^{\mathbb{Z}}, \sigma(w)=$ $\left(a_{i+1}\right)_{i \in \mathbb{Z}}$. Now, let $\sim$ be the equivalence relation on $A^{\mathbb{Z}}$ given by

$$
w \sim z \quad \text { if and only if } \exists n \in \mathbb{Z}, z=\sigma^{n}(w) .
$$

We denote by $\mathcal{O}(w)$ the equivalence class of an element $w \in A^{\mathbb{Z}}$ relative to $\sim$ and we say that $\mathcal{O}(w)$ is the orbit of $w$. It is a straightforward observation that any two $\sim$-equivalent bi-infinite 
words have the same factors. Throughout this paper, when working with a bi-infinite word, we will frequently be interested only on its factors. So we will usually not distinguish a bi-infinite word from its orbit.

It is well known that a bi-infinite word $w$ is periodic if and only if $\mathcal{O}(w)$ is a finite set. For instance, the orbit of the periodic word $w=(a b c a)^{-\infty} \cdot(a b c a)^{+\infty}$ is

$$
\mathcal{O}(w)=\left\{w,(b c a a)^{-\infty} \cdot(b c a a)^{+\infty},(c a a b)^{-\infty} \cdot(c a a b)^{+\infty},(a a b c)^{-\infty} \cdot(a a b c)^{+\infty}\right\} .
$$

Given words $x \in A^{-\mathbb{N}}$ and $y \in A^{\mathbb{N}}$, we denote by $x y$ the orbit of the bi-infinite word $x \cdot y$. For $u \in A^{+}$, we denote $u^{\infty}=u^{-\infty} u^{+\infty}$. For instance, $(a b c a)^{\infty}$ represents the orbit $\mathcal{O}(w)$ in $(2.1)$.

\subsection{Pseudovarieties, implicit operations and implicit signatures}

Given a semigroup $S$, we let $S^{1}$ be the semigroup $S$ itself if it is a monoid, or the disjoint union $S \uplus\{1\}$ where 1 acts as a neutral element otherwise. Given an element $s$ of a compact topological semigroup, the closed subsemigroup generated by $s$ contains a unique idempotent, denoted $s^{\omega}$. Moreover, $s^{\omega-1}$ denotes the inverse of $s^{\omega+1}\left(=s^{\omega} s\right)$ in the maximal closed subgroup containing $s^{\omega}$.

For a pseudovariety $\mathbf{V}$ of semigroups, we denote by $\bar{\Omega}_{A} \mathbf{V}$ the pro- $\mathbf{V}$ semigroup freely generated by $A$, which means that, for each pro-V semigroup $S$ and each function $\varphi: A \longrightarrow S$, there is a unique continuous homomorphism $\bar{\varphi}: \bar{\Omega}_{A} \mathbf{V} \longrightarrow S$ extending $\varphi$. Elements of $\bar{\Omega}_{A} \mathbf{V}$ are called pseudowords over $\mathbf{V}$. Each pseudoword $\pi$ from $\bar{\Omega}_{A} \mathbf{V}$ has a natural interpretation as an (A-ary) implicit operation: to each pro- $\mathbf{V}$ semigroup $S$ is associated an operation $\pi_{S}: S^{A} \longrightarrow S$ which maps $\varphi \in S^{A}$ to $\bar{\varphi}(\pi)$. For instance, for $A=\{a, b\}$, the pseudoword $\pi=a b$ is interpreted as the semigroup multiplication from $S \times S$ into $S$. If $A=\{a\}$, the interpretations of the $\omega$-power $a^{\omega}$ and of the $(\omega-1)$-power $a^{\omega-1}$, are respectively the mappings which associate $s^{\omega}$ and $s^{\omega-1}$ to each element $s \in S$. The subsemigroup of $\bar{\Omega}_{A} \mathbf{V}$ generated by $A$, denoted by $\Omega_{A} \mathbf{V}$, is a dense subsemigroup of $\bar{\Omega}_{A} \mathbf{V}$ whose elements are said to be finite pseudowords or explicit operations over $\mathbf{V}$.

We denote by $\mathbf{S}$ the pseudovariety of all finite semigroups and, for each pseudovariety $\mathbf{V}$, $p_{\mathbf{V}}$ denotes the canonical projection from $\bar{\Omega}_{A} \mathbf{S}$ into $\bar{\Omega}_{A} \mathbf{V}$. By definition, a pseudoidentity is a formal equality $\pi=\rho$, with $\pi, \rho \in \bar{\Omega}_{A} \mathbf{S}$ for some alphabet $A$. When $\pi, \rho \in \Omega_{A} \mathbf{S}, \pi=\rho$ is also called an identity. A finite semigroup $S$ satisfies a pseudoidentity $\pi=\rho$ if $\pi_{S}=\rho_{S}$. We say that a pseudovariety $\mathbf{V}$ satisfies a pseudoidentity $\pi=\rho$, written $\mathbf{V}=\pi=\rho$, if every semigroup in $\mathbf{V}$ satisfies $\pi=\rho$, which means that $p_{\mathbf{V}}(\pi)=p_{\mathbf{V}}(\rho)$. By Reiterman's theorem [21], each pseudovariety is defined by a set $\Sigma$ of pseudoidentities. The pseudovariety defined by $\Sigma$ is denoted by $\llbracket \Sigma \rrbracket$. Here are definitions by pseudoidentities of some pseudovarieties of interest in this paper:

- $\mathbf{D}=\llbracket b a^{\omega}=a^{\omega} \rrbracket$ and $\mathbf{K}=\llbracket a^{\omega} b=a^{\omega} \rrbracket$, the classes of all finite semigroups whose idempotents are right zeros and left zeros, respectively.

- $\mathbf{L I}=\llbracket a^{\omega} b c^{\omega}=a^{\omega} c^{\omega} \rrbracket$ and $\mathbf{L S} \mathbf{S}=\llbracket a^{\omega} b a^{\omega} b a^{\omega}=a^{\omega} b a^{\omega}, a^{\omega} b a^{\omega} c a^{\omega}=a^{\omega} c a^{\omega} b a^{\omega} \rrbracket$, the classes of all finite semigroups $S$ such that, for all idempotents $e \in S, e S e=e$ and $e S e$ is a semilattice, respectively. 
- $\mathbf{G}=\llbracket a^{\omega} b=b a^{\omega}=b \rrbracket$ and $\mathbf{C R}=\llbracket a^{\omega+1}=a \rrbracket$, the classes of all finite groups and completely regular semigroups, respectively.

Given $\pi \in \bar{\Omega}_{A} \mathbf{S}$, we say that $\rho \in \bar{\Omega}_{A} \mathbf{S}$ is a factor (resp. a prefix, a suffix) of $\pi$ if there are $\pi_{1}, \pi_{2} \in\left(\bar{\Omega}_{A} \mathbf{S}\right)^{1}$ such that $\pi=\pi_{1} \rho \pi_{2}$ (resp. $\pi=\rho \pi_{2}, \pi=\pi_{1} \rho$ ). A bi-infinite word $w$ is a (bi-infinite) factor of $\pi$ if every finite word which is a factor of $w$ is also a factor of $\pi$. Notice that, a bi-infinite word $w$ is a factor of $\pi$ if and only if every element of $\mathcal{O}(w)$ is a factor of $\pi$.

An implicit signature $\sigma$ is a set of pseudowords containing the multiplication. It is nontrivial if it contains at least a pseudoword which is not a word. In particular, we denote by $\kappa$ the most commonly used implicit signature $\left\{a b, a^{\omega-1}\right\}$, usually called the canonical signature. Every profinite semigroup has a natural structure of a $\sigma$-semigroup, via the interpretation of implicit operations as operations on profinite semigroups. Let $T_{A}^{\sigma}$ denote the free $\sigma$-algebra generated by $A$ in the variety defined by the identity $x(y z)=(x y) z$, whose elements are called $\sigma$-semigroups. The elements of $T_{A}^{\sigma}$ will be called $\sigma$-terms.

For a pseudovariety $\mathbf{V}$, we denote by $\Omega_{A}^{\sigma} \mathbf{V}$ the free $\sigma$-semigroup generated by $A$ in the variety of $\sigma$-semigroups generated by $\mathbf{V}$, which is the $\sigma$-subsemigroup of $\bar{\Omega}_{A} \mathbf{V}$ generated by $A$. Elements of $\Omega_{A}^{\sigma} \mathbf{V}$ are called $\sigma$-words over $\mathbf{V}$, and $\sigma$-words over $\mathbf{S}$ will be simply referred as $\sigma$-words. We denote by $\varepsilon_{A, \mathbf{V}}^{\sigma}$ the homomorphism of $\sigma$-semigroups $T_{A}^{\sigma} \rightarrow \Omega_{A}^{\sigma} \mathbf{V}$ that sends each letter $a \in A$ to itself. The $\sigma$-word problem for $\mathbf{V}$ consists in determining whether two given $\sigma$-terms $x, y \in T_{A}^{\sigma}$ represent the same element of $\Omega_{A}^{\sigma} \mathbf{V}$, that is, whether $\varepsilon_{A, \mathbf{V}}^{\sigma}(x)=\varepsilon_{A, \mathbf{V}}^{\sigma}(y)$.

For simplicity of notation, we will sometimes not distinguish a $\sigma$-term $x \in T_{A}^{\sigma}$ from the corresponding $\sigma$-word $\varepsilon_{A, \mathbf{S}}^{\sigma}(x) \in \Omega_{A}^{\sigma} \mathbf{S}$. For instance, given two $\sigma$-terms $x, y \in T_{A}^{\sigma}$, when we say " $\mathbf{V} \models x=y$ ", we mean "V $\models \varepsilon_{A, \mathbf{S}}^{\sigma}(x)=\varepsilon_{A, \mathbf{S}}^{\sigma}(y)$ ".

\subsection{Graphs, $\sigma$-reducibility and $\sigma$-tameness}

A (directed multi)graph $\Gamma$ is a disjoint union $\mathcal{V}(\Gamma) \uplus \mathcal{E}(\Gamma)$, with vertex set $\mathcal{V}(\Gamma)$, edge set $\mathcal{E}(\Gamma)$, and edges $\alpha(\mathrm{e}) \stackrel{\mathrm{e}}{\rightarrow} \omega(\mathrm{e})$. The vertices $\alpha(\mathrm{e})$ and $\omega(\mathrm{e})$ are, respectively, the beginning and the end of edge e. A path of $\Gamma$ is a sequence $e_{1}, \ldots, e_{m}$ of edges of $\Gamma$ such that $\omega\left(\mathrm{e}_{i}\right)=\alpha\left(\mathrm{e}_{i+1}\right)$ for $1 \leq i \leq m-1$, and it is a circuit if $\alpha\left(\mathrm{e}_{1}\right)=\omega\left(\mathrm{e}_{m}\right)$. A sequence $\mathrm{e}_{1}, \ldots, \mathrm{e}_{m}$ is said to be a non-oriented path if it is possible to invert the orientation of some edges in such a way as to obtain a path. A labeling of a graph $\Gamma$ by a semigroup $S$ is a mapping $\delta$ from $\Gamma$ into $S^{1}$ such that $\delta(\mathcal{E}(\Gamma)) \subseteq S$. If $\gamma: \mathcal{E}(\Gamma) \longrightarrow A^{+}$is a function, the label of a non-oriented path $\mathrm{e}_{1}, \ldots, \mathrm{e}_{m}$ is the reduced form of the word $\gamma\left(\mathrm{e}_{1}\right)^{\epsilon_{1}} \cdots \gamma\left(\mathrm{e}_{m}\right)^{\epsilon_{m}}$ in the free group generated by $A$, where $\epsilon_{i}=1$ if in the non-oriented path the edge $\mathrm{e}_{i}$ is read in the direct way and $\epsilon_{i}=-1$ otherwise. We say that the function $\gamma$ commutes if the label of any non-oriented circuit is 1 . If $\gamma$ is the restriction to edges of a labeling $\delta$ of $\Gamma$, then we also say that $\delta$ commutes if $\gamma$ commutes. We associate to a finite graph $\Gamma$ a system of equations $\Sigma_{\Gamma}$, with variable set $\Gamma$, formed by all equations of the form $\alpha(\mathrm{e}) \mathrm{e}=\omega(\mathrm{e})$ with $\mathrm{e} \in \mathcal{E}(\Gamma)$.

Let $\mathbf{V}$ be a pseudovariety of semigroups, $S$ be a finite $A$-generated semigroup, $\psi: \bar{\Omega}_{A} \mathbf{S} \rightarrow S$ be a continuous homomorphism respecting the choice of generators and $\gamma: \Gamma \rightarrow S$ be a mapping. We say that a mapping $\delta: \Gamma \rightarrow \bar{\Omega}_{A} \mathbf{S}$ is a solution of $\Sigma_{\Gamma}$ over $\mathbf{V}$ with respect to $(\gamma, \psi)$ if $\psi \circ \delta=\gamma$ and $\mathbf{V}$ satisfies $\delta(\alpha(\mathrm{e})) \delta(\mathrm{e})=\delta(\omega(\mathrm{e}))$ for all $\mathrm{e} \in \mathcal{E}(\Gamma)$. Furthermore, if $\sigma$ is an implicit signature such that $\delta(\Gamma) \subseteq \Omega_{A}^{\sigma} \mathbf{S}$, then $\delta$ is called a $\sigma$-solution of $\Sigma_{\Gamma}$ over $\mathbf{V}$ with 
respect to $(\gamma, \psi)$. The pair $(\gamma, \psi)$ will be sometimes understood and, by abuse of language, we sometimes speak of "solution of $\Gamma$ " instead of "solution of $\Sigma_{\Gamma}$ ".

A pseudovariety $\mathbf{V}$ is said to be $\sigma$-reducible for $\Sigma_{\Gamma}$ if the existence of a solution $\delta$ of $\Sigma_{\Gamma}$ over $\mathbf{V}$ with respect to $(\gamma, \psi)$ entails the existence of a $\sigma$-solution $\delta^{\prime}$ of $\Sigma_{\Gamma}$ over $\mathbf{V}$ with respect to the same pair $(\gamma, \psi)$. If $\mathbf{V}$ is $\sigma$-reducible for $\Sigma_{\Gamma}$ for every finite graph $\Gamma$, then we say that $\mathbf{V}$ is $\sigma$-reducible. A pseudovariety $\mathbf{V}$ is said to be $\sigma$-tame if it is recursively enumerable, $\sigma$-reducible and the $\sigma$-word problem for $\mathbf{V}$ is decidable. Finally, we say that a pseudovariety is tame if it is $\sigma$-tame with respect to a recursively enumerable implicit signature $\sigma$ consisting of computable implicit operations.

The following useful lemma is proved in [15]. To be more precise, condition (a) of the lemma was only proved for edges but its extension to vertices may be proved similarly.

Lemma 2.1 Let $\mathbf{V}$ be a $\sigma$-reducible pseudovariety for a non-trivial implicit signature $\sigma$ and let $\delta: \Gamma \rightarrow \bar{\Omega}_{A} \mathbf{S}$ be a solution of a finite graph $\Gamma$ over $\mathbf{V}$ with respect to $(\gamma, \psi)$. There is a $\sigma$-solution $\delta^{\prime}$ of $\Gamma$ over $\mathbf{V}$ with respect to $(\gamma, \psi)$ that verifies the following conditions, for every $\mathrm{g} \in \Gamma:$

(a) if $\delta(\mathrm{g})$ is an infinite pseudoword, then $\delta^{\prime}(\mathrm{g})$ is an infinite $\sigma$-word;

(b) if $\delta(\mathrm{g})$ is a finite word, then $\delta^{\prime}(\mathrm{g})=\delta(\mathrm{g})$.

See [5, Proposition 3.3] for an extension of this lemma. In particular, as observed in that paper, the following remark holds.

Remark 2.2 Assume the conditions of Lemma 2.1. We can constrain the values under $\delta^{\prime}$ of each $\mathrm{g} \in \Gamma$ with respect to properties which, as that of (b), can be tested in a finite semigroup.

See also [5, Subsection 3.2] for a detailed explanation of how Lemma 2.1 (or its extension) and Remark 2.2 can be used to prove reducibility of joins. We will follow that technique in Section 5 below to obtain a reduction for the problem of the $\kappa$-reducibility of $\mathbf{L S l} \vee \mathbf{V}$.

\section{Implicit operations on LSl}

This section gathers some basic statements about free pro-LSl semigroups.

\subsection{Basic properties of LSl}

We begin by recalling that the pseudovariety LSl is associated via Eilenberg's correspondence with the class of locally testable languages, as shown independently by Brzozowski and Simon [13] and McNaughton [20]. There is a concept of locally testable semigroup, studied by Zalcstein in [25], which is similar to that of locally testable language. A semigroup $S$ is locally testable if it is $k$-testable for some $k>0$, which means that, if two words over the alphabet $S$ have the same set of factors of length $k$, the same prefix and the same suffix of length $k-1$, then the products in $S$ determined by these words are equal. Denote by LT the class of all locally testable semigroups and by $\mathbf{L T}_{k}$ the class of all $k$-testable semigroups. By Zalcstein's results, we know that $\mathbf{L T}$ and $\mathbf{L} \mathbf{T}_{k}$ are pseudovarieties and that $\mathbf{L T}$ is precisely $\mathbf{L S l}$. So, $\mathbf{L T}_{k}$ is also denoted by $\mathbf{L S l}_{k}$. 
For a word $w \in A^{+}$, denote by $F_{k}(w)$ the set of all factors of $w$ of length $k$ and denote by $i_{k}(w)$ (resp. $\left.t_{k}(w)\right)$ the word $w$ if $|w|<k$ and the word $p_{k}(w)$ (resp. $s_{k}(w)$ ) otherwise. The relation $\sim_{k}$ over $A^{+}$defined by

$$
u \sim_{k} v \quad \text { if } \quad i_{k-1}(u)=i_{k-1}(v), t_{k-1}(u)=t_{k-1}(v) \text { and } F_{k}(u)=F_{k}(v),
$$

is a finite index congruence. This means that the quotient $A^{+} / \sim_{k}$ is a finite semigroup. As a consequence, we have the following characterization of the free pro- $\mathbf{L} \mathbf{T}_{k}$ semigroups.

Proposition 3.1 For all $k \geq 1, \bar{\Omega}_{A} \mathbf{L} \mathbf{T}_{k}=\Omega_{A} \mathbf{L} \mathbf{T}_{k}=A^{+} / \sim_{k}$.

We next notice that $\mathbf{K}, \mathbf{D}$ and $\mathbf{L I}$ are important subpseudovarieties of $\mathbf{L S I}$ and that $\mathbf{L I}$ is the join of $\mathbf{K}$ and $\mathbf{D}$. The last observation means that a pseudoidentity $\pi=\rho$ is satisfied by $\mathbf{L I}$ if and only if is satisfied by both $\mathbf{K}$ and $\mathbf{D}$. Recall that if $\mathbf{V}$ is one of the pseudovarieties $\mathbf{K}, \mathbf{D}, \mathbf{L I}$ or $\mathbf{L S l}$, then it does not satisfy any non-trivial identity, whence we may identify the subsemigroup $\Omega_{A} \mathbf{V}$ of $\bar{\Omega}_{A} \mathbf{V}$ with $A^{+}$. Moreover, $\bar{\Omega}_{A} \mathbf{K}$ and $\bar{\Omega}_{A} \mathbf{D}$ are isomorphic to $A^{\infty}$ and $A^{-\infty}$, respectively. In $\bar{\Omega}_{A} \mathbf{K}$, the right-infinite word $v u^{+\infty}$, where $v \in A^{*}$ and $u \in A^{+}$, corresponds to the pseudoword $v u^{\omega}$. A dual remark holds for $\mathbf{D}$.

Let $n$ be a positive integer. We denote by $\equiv_{n}$ the congruence on $\bar{\Omega}_{A} \mathbf{S}$ given, for every $\pi, \rho \in \bar{\Omega}_{A} \mathbf{S}$, by

$$
\pi \equiv_{n} \rho \quad \text { if } \quad \pi \text { and } \rho \text { have the same prefix, suffix and factors of length } n .
$$

The following proposition characterizes pseudoidentities satisfied by LSl. It is an immediate consequence of [14, Theorem 3.3].

Proposition 3.2 Let $\pi, \rho \in \bar{\Omega}_{A} \mathbf{S}$. The following conditions are equivalent:
i) $p_{\mathbf{L S I}}(\pi)=p_{\mathbf{L S I}}(\rho)$;
ii) $\pi \equiv_{n} \rho$ for every $n \in \mathbb{N}$;
iii) $p_{\mathbf{L I}}(\pi)=p_{\mathbf{L I}}(\rho)$ and $\pi$ and $\rho$ have the same finite factors;
iv) $p_{\mathbf{L I}}(\pi)=p_{\mathbf{L I}}(\rho)$ and $\pi$ and $\rho$ have the same bi-infinite factors.

Moreover, if $\pi$ and $\rho$ are infinite pseudowords, then a bi-infinite word $\mathbf{w} \in A^{\mathbb{Z}}$ is a factor of $\pi \rho$ if and only if $\mathbf{w}$ is a factor of $\pi$ or a factor of $\rho$, or $\mathbf{w} \in \mathcal{O}\left(p_{\mathbf{D}}(\pi) \cdot p_{\mathbf{K}}(\rho)\right)$.

\subsection{The $\kappa$-word problem for LSl}

We now briefly recall the solution of the $\kappa$-word problem for LSl [14]. For the rest of the paper, the homomorphism of $\kappa$-semigroups $\varepsilon_{A, \mathbf{S}}^{\kappa}: T_{A}^{\kappa} \rightarrow \Omega_{A}^{\kappa} \mathbf{S}$ will be simply denoted by $\varepsilon$. To simplify the notation, the homomorphism $\varepsilon$ will be sometimes omitted: when we refer to a $\kappa$-term $x \in T_{A}^{\kappa}$, we want to consider in those cases the corresponding $\kappa$-word $\varepsilon(x) \in \Omega_{A}^{\kappa} \mathbf{S}$.

Let $x \in \Omega_{A}^{\kappa} \mathbf{S}$ be a $\kappa$-word. Notice that $x^{\omega-1} x=x^{\omega}$ since, for each element $s$ of a finite semigroup, $s^{\omega-1} s=s^{\omega-1} s^{\omega} s=s^{\omega-1} s^{\omega+1}=s^{\omega}$ by definition of $s^{\omega}$ and $s^{\omega-1}$. For each integer $i$, we let

$$
x^{\omega+i}= \begin{cases}\left(x^{\omega-1}\right)^{-i} & \text { if } i<0 \\ x^{\omega} & \text { if } i=0 \\ x^{\omega} x^{i} & \text { if } i>0 .\end{cases}
$$


The following identities of $\kappa$-words, where $x, y \in \Omega_{A}^{\kappa} \mathbf{S}$ and $i, j \in \mathbb{Z}$, are easily established

$$
\begin{aligned}
& \left(x^{\omega+i}\right)^{\omega+j}=x^{\omega+i j}, \\
& \left(x^{i}\right)^{\omega+j}=x^{\omega+i j}, \quad(i>0) \\
& x^{\omega+i} x^{\omega+j}=x^{\omega+i+j}, \\
& x^{i} x^{\omega+j}=x^{\omega+j} x^{i}=x^{\omega+i+j}, \quad(i>0) \\
& (x y)^{\omega+i}=x(y x)^{\omega+i-1} y .
\end{aligned}
$$

We adopt the notation $x^{\omega+i}$ also for $\kappa$-terms and denote $\{\omega+i \mid i \in \mathbb{Z}\}$, called the set of infinite exponents, by $\omega+\mathbb{Z}$. The rank of a $\kappa$-term is the maximum number of nested infinite powers in it. For instance, the expression

$$
a\left(a(b a)^{\omega-2} b\right)^{\omega+3} b^{4}\left(b\left(a^{3}\right)^{\omega-1} a\left(a^{2}\right)^{\omega}\right)^{\omega+1} a
$$

represents a $\kappa$-term $w$ of rank 2 on the alphabet $\{a, b\}$. So, a $\kappa$-term of rank 0 is simply a word from $A^{+}$. A $\kappa$-term of rank 1 is an element $w \in T_{A}^{\kappa}$ of the form

$$
w=u_{0}^{\prime} u_{1}^{\alpha_{1}} u_{1}^{\prime} u_{2}^{\alpha_{2}} \cdots u_{m}^{\alpha_{m}} u_{m}^{\prime}
$$

with $m \geq 1, u_{0}^{\prime}, \ldots, u_{m}^{\prime} \in A^{*}, u_{1}, \ldots, u_{m} \in A^{+}$and $\alpha_{1}, \ldots, \alpha_{m} \in \omega+\mathbb{Z}$. It is clear that $\varepsilon_{A, \mathbf{K}}^{\kappa}(w)$ is the right-infinite word $u_{0}^{\prime} u_{1}^{+\infty}$ while $\varepsilon_{A, \mathbf{D}}^{\kappa}(w)$ is the left-infinite word $u_{m}^{-\infty} u_{m}^{\prime}$. The $\kappa$-term $w$ is said to be in reduced form when $u_{1}, \ldots, u_{m}$ are Lyndon words and $u_{j}^{-\infty} u_{j}^{\prime} u_{j+1}^{+\infty}$ is a non-periodic bi-infinite word for $j=1, \ldots, m-1$.

For a $\kappa$-term $w$, we denote by $B_{w}$ the set of non-periodic bi-infinite factors of the $\kappa$-word $\varepsilon(w)$. In case $w=u_{0}^{\prime} u_{1}^{\alpha_{1}} u_{1}^{\prime} u_{2}^{\alpha_{2}} \cdots u_{m}^{\alpha_{m}} u_{m}^{\prime}$ is a rank $1 \kappa$-term written in reduced form, then it is immediate that $B_{w}$ is the following set of bi-infinite words

$$
B_{w}=\left\{u_{j}^{-\infty} u_{j}^{\prime} u_{j+1}^{+\infty} \mid j=1, \ldots, m-1\right\} .
$$

Notice that since $\mathbf{L S l}$ is an aperiodic pseudovariety, it verifies the pseudoidentity $x^{\omega+i}=x^{\omega}$ for every integer $i$. We also recall the following well-known property which permits to reduce the $\kappa$-word problem for LSl to identities involving only $\kappa$-terms of rank at most 1 .

Lemma 3.3 If $w \in T_{A}^{\kappa} \backslash A^{+}$, then $\mathbf{L S l} \models w^{\omega+i}=w^{2}$ for every integer $i$.

Notice at last that, if a pseudoidentity $\pi=\rho$ holds in LSl, then either $\pi$ and $\rho$ are the same finite word or they are both infinite pseudowords. The following decision criterion to test whether two infinite $\kappa$-terms (i.e., $\kappa$-terms of rank at least 1 ) are equal over LSl is a simple reformulation of $[14$, Theorem 7.1].

Proposition 3.4 Let $w \in T_{A}^{\kappa}$ be an infinite $\kappa$-term. Then, there is a rank $1 \kappa$-term $w_{1}=$ $u_{0}^{\prime} u_{1}^{\omega} u_{1}^{\prime} u_{2}^{\omega} \cdots u_{m}^{\omega} u_{m}^{\prime}$ in reduced form such that $\mathbf{L S 1} \models w=w_{1}$.

Moreover, if $z \in T_{A}^{\kappa}$ is another infinite $\kappa$-term and $z_{1}=v_{0}^{\prime} v_{1}^{\omega} v_{1}^{\prime} v_{2}^{\omega} \cdots v_{n}^{\omega} v_{n}^{\prime}$ is a rank $1 \kappa$ term in reduced form such that $\mathbf{L S} 1=z=z_{1}$, then $\mathbf{L S} \mid=w=z$ if and only if $u_{0}^{\prime} u_{1}^{+\infty}=v_{0}^{\prime} v_{1}^{+\infty}$, $u_{m}^{-\infty} u_{m}^{\prime}=v_{n}^{-\infty} v_{n}^{\prime}$ and $B_{w_{1}}=B_{z_{1}}$. Furthermore, it is effectively decidable whether $\mathbf{L S} 1 \models w=z$. 
Let $w \in T_{A}^{\kappa}$ be an infinite $\kappa$-term. As a consequence of Proposition 3.2, we have $B_{w}=B_{w_{1}}$, where $w_{1}$ is any rank $1 \kappa$-term in reduced form such that LSl $\models w=w_{1}$. For example, suppose that $w=a\left(a(b a)^{\omega-2} b\right)^{\omega+3} b^{4}\left(b\left(a^{3}\right)^{\omega-1} a\left(a^{2}\right)^{\omega}\right)^{\omega+1} a$ is the $\kappa$-term in (3.6) and assume that $a<b$. The pseudovariety $\mathbf{L S l}$ verifies the following $\kappa$-identities

$$
\begin{aligned}
w & =a\left(a b(a b)^{\omega-3} a b\right)^{\omega+3} b^{4}\left(b a^{\omega-3} a a^{\omega}\right)^{\omega+1} a & & \text { by (3.2) and (3.5) } \\
& =a\left((a b)^{\omega-1}\right)^{\omega+3} b^{4}\left(b a^{\omega-2}\right)^{\omega+1} a & & \text { by (3.3) and (3.4) } \\
& =a(a b)^{\omega-3} b^{4} b a^{\omega-2} b a^{\omega-2} a & & \text { by (3.1) and Lemma } 3.3 \\
& =a(a b)^{\omega} b^{5} a^{\omega} b a^{\omega} & & \text { by (3.4) and since LSl } \models x^{\omega+i}=x^{\omega} .
\end{aligned}
$$

As this $\kappa$-term is in reduced form, we deduce that $B_{w}=\left\{(a b)^{-\infty} b^{5} a^{+\infty}, a^{-\infty} b a^{+\infty}\right\}$.

\section{Some combinatorial results}

In this section, we recall some definitions and results on words, introduced in [17, Section 4], that will be used latter. The reader is referred to that paper for further details.

For the rest of the paper, when we refer to a solution of a certain graph $\Gamma$ over a certain pseudovariety with respect to a pair $(\gamma, \psi)$, we assume that $\gamma: \Gamma \rightarrow S$ and $\psi: \bar{\Omega}_{A} \mathbf{S} \rightarrow S$ are mappings into a certain fixed finite $A$-generated semigroup $S$.

\subsection{Marked factors}

We begin by fixing several integers, already used in [17]:

$$
k=|S|+2 ; \quad k^{\prime}=6 k|A|^{3 k} ; \quad k^{\prime \prime}=\left[2 k^{\prime}(3 k-1)\right]^{|A|^{3 k-1}+1} .
$$

A finite word $v$ is said to be $k^{\prime}$-abundant if $\operatorname{docc}(y, v) \geq k^{\prime}$ for all factors $y$ of $v$ with length $3 k-1$. Let $w=a_{1} a_{2} \cdots a_{m}\left(a_{i} \in A\right)$ be a finite word, with $m \geq 3 k-1$. A $k^{\prime \prime}$-neighborhood of an occurrence $u=w[i, j]$ of a factor $u$ in $w$ is an occurrence $v=w\left[i^{\prime}, j^{\prime}\right]$ extending $w[i, j]$ (i.e., such that $i^{\prime} \leq i$ and $j^{\prime} \geq j$ ) and such that $|v| \leq k^{\prime \prime}$. An occurrence $u=w[i, j]$, with $|u|=3 k-1$, will be said to be free if there exists a $k^{\prime \prime}$-neighborhood $v$ of $w[i, j]$ such that $v$ is $k^{\prime}$-abundant. Therefore, in this case, every occurrence of a factor $y$ of length $3 k-1$ in $v$, is free. The occurrence $w[i, j]$ (and the letters $a_{i}, a_{i+1}, \ldots, a_{j}$ ) will be said to be marked if it is not free.

Lemma 4.1 There is a unique factorization $w=w_{0} v_{1} w_{1} v_{2} \cdots v_{q} w_{q}$ such that $q \geq 0$ and

- $w_{0}, w_{q} \in A^{*}, w_{1}, \ldots, w_{q-1}, v_{1}, \ldots, v_{q} \in A^{+}$;

- for each $1 \leq i \leq q$, the letters of $v_{i}$ are marked;

- for each $0 \leq j \leq q$, the letters of $w_{j}$ are not marked.

This factorization is called the marked factorization of $w$ (for $k$ ). The factors $v_{1}, \ldots, v_{q}$ and $w_{0}, \ldots, w_{q}$ are said to be, respectively, the marked factors and the free factors of $w$ (for $k$ ). 


\subsection{Transforming words into rank $1 \kappa$-terms}

In [17] it is defined a function that associates to certain finite words $u$ certain rank $1 \kappa$ terms $\bar{u}$. Here, we make a small adjustment on that definition and notice that, as far as the pseudovariety LSl is concerned, that modification is harmless (in the sense that the new version could substitute the old one so that the results in [17] would still hold).

By the pigeonhole principle, for each word $u=a_{1} \cdots a_{k} \in A^{+}$of length $k=|S|+2$, there exist indices $i$ and $j$ such that $1<i \leq j<k$ and $S \models a_{1} \cdots a_{i-1}=a_{1} \cdots a_{j}$. As a consequence, $S$ verifies $a_{1} \cdots a_{j}=a_{1} \cdots a_{i-1}\left(a_{i} \cdots a_{j}\right)^{m}$ for every positive integer $m$, whence

$$
S \models u=a_{1} \cdots a_{i-1}\left(a_{i} \cdots a_{j}\right)^{\omega+1} a_{j+1} \cdots a_{k} .
$$

Suppose that $a_{i} \cdots a_{j}$ is not a primitive word. Then $a_{i} \cdots a_{j}=\left(a_{i} \cdots a_{\ell}\right)^{n}$ for some $i \leq \ell<j$ and $n>1$ such that $a_{i} \cdots a_{\ell}$ is a primitive word. Hence, $S$ verifies

$$
\begin{aligned}
u & =a_{1} \cdots a_{i-1}\left(\left(a_{i} \cdots a_{\ell}\right)^{n}\right)^{\omega} a_{i} \cdots a_{j} a_{j+1} \cdots a_{k} \\
& =a_{1} \cdots a_{i-1}\left(a_{i} \cdots a_{\ell}\right)^{\omega} a_{i} \cdots a_{j} a_{j+1} \cdots a_{k} \\
& =a_{1} \cdots a_{i-1}\left(a_{i} \cdots a_{\ell}\right)^{\omega+1} a_{\ell+1} \cdots a_{j} a_{j+1} \cdots a_{k} .
\end{aligned}
$$

Therefore, we may assume without loss of generality that, in (4.1), $a_{i} \cdots a_{j}$ is a primitive word. Now, there exists an integer $i \leq i^{\prime} \leq j$ such that $a_{i^{\prime}} \cdots a_{j} a_{i} \cdots a_{i^{\prime}-1}$ is a Lyndon word. We let $\bar{u}$ be the following $\kappa$-term

$$
\bar{u}=a_{1} \cdots a_{i-1} a_{i} \cdots a_{i^{\prime}-1}\left(a_{i^{\prime}} \cdots a_{j} a_{i} \cdots a_{i^{\prime}-1}\right)^{\omega} a_{i^{\prime}} \cdots a_{j} a_{j+1} \cdots a_{k}
$$

and notice that $S \models u=\bar{u}$ by (3.5). Now, recall that there is a positive integer $n_{S}$, called the exponent of $S$, such that $s^{n_{S}}=s^{\omega}$ for all $s \in S$. Let $m_{S}=p n_{S}+1$ for some $p$ such that $p n_{S}+1>k$, and notice that $s^{m_{S}}=s^{\omega+1}$ for all $s \in S$. So, in particular, if $v \in A^{+}$is any Lyndon word, then the semigroup $S$ satisfies $v^{m_{S}}=v^{\omega+1}$ and we may define

$$
u=v^{m_{S}} \quad \text { and } \quad \bar{u}=u^{\omega+1}=v^{\omega+m_{S}},
$$

without conflict with the previous case since $|u|=m_{S}|v|>k$. Notice that also in this case $S \models u=\bar{u}$. Indeed, $S$ satisfies $u^{\omega+1}=\left(v^{m_{S}}\right)^{\omega} v^{m_{S}}=v^{\omega} v^{\omega+1}=v^{\omega+1}=v^{m_{S}}=u$. Notice furthermore that in both cases, if $\mathbf{V}$ is a pseudovariety that verifies the pseudoidentity $x y^{\omega+1} z=x y z$, then $\mathbf{V}$ also verifies $u=\bar{u}$. Indeed, in case $\bar{u}$ is given by (4.2), it suffices to note that $\mathbf{V}$ satisfies the pseudoidentity (3.5). When $\bar{u}$ is given by (4.3), $\mathbf{V}$ verifies $u=v v^{m_{S}-2} v=$ $v\left(v^{m_{S}-2}\right)^{\omega+1} v=v v^{\omega+m_{S}-2} v=v^{\omega+m_{S}}=\bar{u}$.

\subsection{Centers of bi-infinite words}

Let $w \in A^{+}$and let $u=w[\ell, r]$ be an occurrence of a factor $u$ in $w$. An occurrence $v=w\left[\ell^{\prime}, r\right]$, with $\ell^{\prime} \leq \ell$, of a factor $v$ in $w$ is said to be a left-extension of the occurrence $w[\ell, r]$. In this case, the word $v$ itself is said to be a left-extension (in $w$ ) of the occurrence $w[\ell, r]$.

Let $u \in A^{+}$and let $\overleftarrow{u}$ be a left-extension of $u$. We say that an occurrence $u=w[\ell, r]$ in a word $w \in A^{+}$is allowed in $w$ relative to $\overleftarrow{u}$, if $\overleftarrow{u}$ is a left-extension in $w$ of the occurrence $w[\ell, r]$. For instance, let $u=a b c$ and let $\overleftarrow{u}=a b a a b c$. Then $w=c a b a$ abcaabcabaabcacbc has 
two allowed occurrences of $u$ relative to $\overleftarrow{u}: w[5,7]$ and $w[15,17] ;$ and one occurrence of $u$ not allowed: $w[9,11]$. If $\overleftarrow{u}=a b b a b c$ then $u$ has no allowed occurrences in $w$

Let $\mathbf{w} \in A^{\mathbb{Z}}$ be a bi-infinite word. For every pair of integers $p, q \in \mathbb{N}_{0}$, the factor $\mathbf{w}[-p, q]$ is said to be a center of $\mathbf{w}$. The next result [17, Lemma 4.2] establishes that allowed occurrences of certain centers of bi-infinite words are necessarily disjoint, a property that is essential to our purposes.

Lemma 4.2 Let $B=\left\{\mathbf{w}_{1}, \ldots, \mathbf{w}_{n}\right\}$ be a finite set of non-periodic bi-infinite words such that $\mathbf{w}_{i} \nsim \mathbf{w}_{j}$ for every $i, j \in\{1, \ldots, n\}$ with $i \neq j$. For each $\ell \in\{1, \ldots, n\}$ let also $c_{\ell}=\mathbf{w}_{\ell}\left[-p_{\ell}, q_{\ell}\right]$ be a center of $\mathbf{w}_{\ell}$ with $q_{\ell} \geq Q$ for a fixed $Q$ (depending on $B$ ) chosen sufficiently large.

Then, for each $\ell$ there is a center $\overleftarrow{c_{\ell}}=\mathbf{w}_{\ell}\left[-p_{\ell}^{\prime}, q_{\ell}\right]$ of $\mathbf{w}_{\ell}$ with $p_{\ell} \leq p_{\ell}^{\prime}$ (so that $\overleftarrow{c_{\ell}}$ is a left-extension of $c_{\ell}$ ) such that the following property is verified, for every $i, j \in\{1, \ldots, n\}$ (where $i$ and $j$ may be the same):

any two distinct occurrences of $c_{i}$ and $c_{j}$ in a finite word $w \in A^{+}$, which are allowed relative to $\overleftarrow{c_{i}}$ and $\overleftarrow{c_{j}}$ respectively, are disjoint

\section{$5 \quad \kappa$-reducibility of joins involving LSl}

The remainder of the paper will be devoted to proving the following main result.

Theorem 5.1 If $\mathbf{V} \subseteq \llbracket x y^{\omega+1} z=x y z \rrbracket$ is $\kappa$-reducible, then so is $\mathbf{L S l} \vee \mathbf{V}$.

Since the $\kappa$-word problem is decidable for $\mathbf{L S l} \vee \mathbf{V}$ if it is decidable for both $\mathbf{L S l}$ and $\mathbf{V}$, the following result is an immediate consequence of Proposition 3.4 and Theorem 5.1.

Corollary 5.2 If $\mathbf{V} \subseteq \llbracket x y^{\omega+1} z=x y z \rrbracket$ is $\kappa$-tame, then so is $\mathbf{L S l} \vee \mathbf{V}$.

In particular, it follows from the tameness results already mentioned in the introduction that $\mathbf{L S l} \vee \mathbf{A b}, \mathbf{L S l} \vee \mathbf{G}, \mathbf{L S l} \vee \mathbf{O C R}$ and $\mathbf{L S l} \vee \mathbf{C R}$ are $\kappa$-tame.

\subsection{First type $\kappa$-reducibility}

For the rest of the paper, $\mathbf{V}$ denotes a $\kappa$-reducible pseudovariety verifying the pseudoidentity $x y^{\omega+1} z=x y z$. We begin by reducing the problem to the case in which all vertices are labeled by infinite pseudowords.

Definition 5.3 (FT $\kappa$-reducibility) We say that $\mathbf{L S I} \vee \mathbf{V}$ is FT ("first type") $\kappa$-reducible if, for every integer $M \geq 1$ and every solution $\delta_{*}$ of a finite graph $\Gamma_{*}$ over $\mathbf{L S l} \vee \mathbf{V}$ such that $\delta_{*}(\mathrm{v})$ is infinite for each vertex $\mathrm{v} \in \Gamma_{*}$, there exists a $\kappa$-solution $\delta_{*}^{\prime}=\delta_{*}^{\prime}\left(\Gamma_{*}, \delta_{*}, M\right)$ of $\Gamma_{*}$ over $\mathbf{L S l} \vee \mathbf{V}$ satisfying the following condition, for each vertex $\vee \in \Gamma_{*}$,

(FT) if $\delta_{*}(\mathrm{v})=u \pi$ where $u \in A^{+}$is a word of length $M$ and $\pi \in \bar{\Omega}_{A} \mathbf{S}$, then $\delta_{*}^{\prime}(\mathrm{v})=u \pi^{\prime}$ where $\pi^{\prime} \in \Omega_{A}^{\kappa} \mathbf{S}$ is such that $\psi(\pi)=\psi\left(\pi^{\prime}\right)$.

We show that the $\kappa$-reducibility of $\mathbf{L S I} \vee \mathbf{V}$ is a consequence of its FT $\kappa$-reducibility.

Proposition 5.4 If $\mathbf{L S l} \vee \mathbf{V}$ is FT $\kappa$-reducible, then it is $\kappa$-reducible. 
Proof. Let $\delta$ be a solution of a finite graph $\Gamma$ over $\mathbf{L S I} \vee \mathbf{V}$ with respect to a pair $(\gamma, \psi)$. We construct a new graph $\Gamma_{*}$ and a new solution $\delta_{*}$ in which all vertices are labeled by infinite pseudowords as follows. Drop all vertices $v$ such that $\delta(\mathrm{v})$ is a finite word and drop all edges beginning in $\mathrm{v}$. Let $\mathcal{E}_{\omega}$ be the set of all edges e $\in \Gamma$ such that $\delta(\alpha(\mathrm{e}))$ is a finite word and $\delta(\mathrm{e})$ is an infinite pseudoword. For each $\mathrm{e} \in \mathcal{E}_{\omega}$, we let $\mathrm{v}_{\mathrm{e}}$ be a new vertex and let $\mathrm{v}_{\mathrm{e}} \stackrel{\mathrm{f}_{\mathrm{e}}}{\longrightarrow} \omega(\mathrm{e})$ be a new edge. Since $\delta(\mathrm{e})$ is infinite, we can write $\delta(\mathrm{e})=x_{\mathrm{e}} y_{\mathrm{e}}$ for some infinite pseudowords $x_{\mathrm{e}}$ and $y_{\mathrm{e}}$. Denote by $\Gamma_{*}$ the new graph thus obtained and let $\delta_{*}: \Gamma_{*} \rightarrow \bar{\Omega}_{A} \mathbf{S}$ be the labeling which coincides with $\delta$ on $\Gamma_{*} \cap \Gamma$ and, for each $\mathrm{e} \in \mathcal{E}_{\omega}$, is such that $\delta_{*}\left(\mathrm{v}_{\mathrm{e}}\right)=\delta(\alpha(\mathrm{e})) \cdot x_{\mathrm{e}}$ and $\delta_{*}\left(\mathrm{f}_{\mathrm{e}}\right)=y_{\mathrm{e}}$. Let also $\gamma_{*}: \Gamma_{*} \rightarrow S$ be the labeling of $\Gamma_{*}$ defined by $\gamma_{*}=\psi \circ \delta_{*}$. Since $\delta$ is a solution of $\Gamma$ over $\mathbf{L S l} \vee \mathbf{V}$ with respect to $(\gamma, \psi)$, it is clear that $\delta_{*}$ is a solution of $\Gamma_{*}$ over $\mathbf{L S l} \vee \mathbf{V}$ with respect to $\left(\gamma_{*}, \psi\right)$. Fix an integer $M$ such that $M \geq|\delta(\mathrm{v})|$ for each vertex $\vee \in \Gamma$ with $\delta(v)$ finite.

By hypothesis LSI $\vee \mathbf{V}$ is FT $\kappa$-reducible. Therefore, there exists a $\kappa$-solution $\delta_{*}^{\prime}=$ $\delta_{*}^{\prime}\left(\Gamma_{*}, \delta_{*}, M\right)$ of $\Gamma_{*}$ over $\mathbf{L S l} \vee \mathbf{V}$ with respect to $\left(\gamma_{*}, \psi\right)$ satisfying condition (FT) above. Hence, for each e $\in \mathcal{E}_{\omega}, \delta_{*}^{\prime}\left(\mathrm{v}_{\mathrm{e}}\right)=\delta(\alpha(\mathrm{e})) \cdot x_{\mathrm{e}}^{\prime}$ for some $x_{\mathrm{e}}^{\prime} \in \Omega_{A}^{\kappa} \mathbf{S}$ such that $\psi\left(x_{\mathrm{e}}\right)=\psi\left(x_{\mathrm{e}}^{\prime}\right)$. Then, we let $\delta^{\prime}$ coincide with $\delta_{*}^{\prime}$ on $\Gamma_{*} \cap \Gamma$ and let $\delta^{\prime}(\mathrm{e})=x_{\mathrm{e}}^{\prime} \cdot \delta_{*}^{\prime}\left(\mathrm{f}_{\mathrm{e}}\right)$ for each e $\in \mathcal{E}_{\omega}$. The remaining elements $\mathrm{g}$ of $\Gamma$, that is $\mathrm{g} \in \Gamma \backslash\left(\Gamma_{*} \cup \mathcal{E}_{\omega}\right)$, are labeled under $\delta$ by finite words, and we let $\delta^{\prime}(\mathrm{g})=\delta(\mathrm{g})$. Therefore $\delta^{\prime}$ is clearly a $\kappa$-solution of $\Gamma$ over $\mathbf{L S I} \vee \mathbf{V}$ with respect to $(\gamma, \psi)$.

With the objective of proving the FT $\kappa$-reducibility of $\mathbf{L S l} \vee \mathbf{V}$, we assume that $M$ is a positive integer and that $\delta_{*}$ is a solution of a finite graph $\Gamma_{*}$ over $\mathbf{L S l} \vee \mathbf{V}$ with respect to a pair $\left(\gamma_{*}, \psi\right)$, labeling each vertex of $\Gamma_{*}$ by an infinite pseudoword. We need to construct a $\kappa$-solution $\delta_{*}^{\prime}=\delta_{*}^{\prime}\left(\Gamma_{*}, \delta_{*}, M\right)$ of $\Gamma_{*}$ over $\mathbf{L S l} \vee \mathbf{V}$ satisfying condition $(\mathrm{FT})$.

\subsection{Some notation for the graph and the solution}

Let $\varepsilon_{0}\left(\Gamma_{*}\right)$ be the set of all edges of $\Gamma_{*}$ labeled under $\delta_{*}$ by finite words and let $\Gamma=\Gamma_{*} \backslash \varepsilon_{0}\left(\Gamma_{*}\right)$. Let also $\delta$ and $\gamma$ be respectively the restrictions of $\delta_{*}$ and $\gamma_{*}$ to $\Gamma$, and notice that $\delta$ is a solution of $\Gamma$ over $\mathbf{L S I} \vee \mathbf{V}$ with respect to $(\gamma, \psi)$. In the rest of the paper, we will use several parameters associated to the elements of $\Gamma$. For one such parameter $f$ and $g \in \Gamma$, the value of $\mathrm{g}$ under $f$ will be usually denoted by $f_{\mathrm{g}}$. We begin by recalling some of these parameters which were introduced in [17, Subsection 6.3] and are related to the solution $\delta$.

For each element $\mathrm{g} \in \Gamma$ and each edge $\mathrm{e} \in \Gamma$, we denote

$$
\mathbf{k}_{\mathrm{g}}=p_{\mathbf{K}}(\delta(\mathrm{g})), \quad \mathbf{d}_{\mathrm{g}}=p_{\mathbf{D}}(\delta(\mathrm{g})), \quad \mathbf{w}_{\mathrm{e}}=\mathbf{d}_{\alpha(\mathrm{e})} \cdot \mathbf{k}_{\mathrm{e}},
$$

and notice that $\mathbf{k}_{\mathrm{g}} \in A^{\mathbb{N}}, \mathbf{d}_{\mathrm{g}} \in A^{-\mathbb{N}}$ and $\mathbf{w}_{\mathrm{e}} \in A^{\mathbb{Z}}$. Moreover, since $\delta$ is a solution of $\Gamma$ over $\mathbf{K}$ and over $\mathbf{D}$, we have the equalities $\mathbf{k}_{\mathrm{v}}=\mathbf{k}_{\mathrm{w}}$ and $\mathbf{d}_{\mathrm{e}}=\mathbf{d}_{\omega(\mathrm{e})}$ for all vertices $\mathrm{v}$ and $\mathbf{w}$ in the same connected component of $\Gamma$ and all edges e.

For each vertex $v$ and each edge e, let

$$
\begin{aligned}
& l_{\mathrm{v}}=\mathbf{k}_{\mathrm{v}}[1, M+k], \quad \quad r_{\mathrm{v}}=\mathbf{d}_{\mathrm{v}}\left[-i_{\mathrm{v}},-1\right], \\
& l_{\mathrm{e}}=\mathbf{k}_{\mathrm{e}}\left[1, i_{\mathrm{e}}\right], \quad r_{\mathrm{e}}=r_{\omega(\mathrm{e})}, \quad c_{\mathrm{e}}=r_{\alpha(\mathrm{e})} l_{\mathrm{e}},
\end{aligned}
$$


where $i_{\mathrm{v}}, i_{\mathrm{e}} \geq M+k$ are integers fixed in Definition 5.5 below. Notice that $l_{\mathrm{g}}, r_{\mathrm{g}}, c_{\mathrm{e}} \in A^{+}$ and that the word $c_{\mathrm{e}}=\mathbf{w}_{\mathrm{e}}\left[-i_{\alpha(\mathrm{e})}, i_{\mathrm{e}}\right.$ [ is a center of the bi-infinite word $\mathbf{w}_{\mathrm{e}}$. Let $F_{\Gamma}$ be a set of edges of $\Gamma$ and let

$$
\begin{array}{ll}
W_{\mathcal{E}}=\left\{\mathbf{w}_{\mathrm{e}}: \mathrm{e} \in \mathcal{E}(\Gamma)\right\}, & C_{\mathcal{E}}=\left\{c_{\mathrm{e}}: \mathrm{e} \in \mathcal{E}(\Gamma)\right\}, \\
W_{F}=\left\{\mathbf{w}_{\mathrm{f}}: \mathrm{f} \in F_{\Gamma}\right\}, & C_{F}=\left\{c_{\mathrm{f}}: \mathrm{f} \in F_{\Gamma}\right\},
\end{array}
$$

with $F_{\Gamma}$ chosen so that $W_{F}$ contains exactly one representative of each orbit $\mathcal{O}\left(\mathbf{w}_{\mathbf{e}}\right)$ with $\mathrm{e} \in \mathcal{E}(\Gamma)$. The set $C_{\mathcal{E}}$ will be called the set of centers of $\mathcal{E}(\Gamma)$, while $C_{F}$ will be called the set of centers of $F_{\Gamma}$. A center $c_{\mathrm{e}}$ is said to be periodic when the bi-infinite word $\mathbf{w}_{\mathrm{e}}$ is periodic.

Let $\mathrm{g} \in \Gamma$. We factorize $l_{\mathrm{g}}$ and $r_{\mathrm{g}}$, and define rank $1 \kappa$-terms $\widehat{l_{\mathrm{g}}}$ and $\widehat{r_{\mathrm{g}}}$, as follows

$$
\begin{aligned}
l_{\mathrm{g}}=l_{\mathrm{g}, 1} l_{\mathrm{g}, 2}, & r_{\mathrm{g}}=r_{\mathrm{g}, 2} r_{\mathrm{g}, 1}, \\
\widehat{l_{\mathrm{g}}}=l_{\mathrm{g}, 1} \overline{l_{\mathrm{g}, 2}}, & \widehat{r_{\mathrm{g}}}=\overline{r_{\mathrm{g}, 2}} r_{\mathrm{g}, 1},
\end{aligned}
$$

where:

$h .1)$ if $\mathrm{g}$ is a vertex or $\mathbf{k}_{\mathrm{g}}$ is non-ultimately periodic, then $l_{\mathrm{g}, 2}$ is the suffix of $l_{\mathrm{g}}$ of length $k$ and $\overline{l_{\mathrm{g}, 2}}$ is given by $(4.2)$;

$h .2)$ if $\mathrm{g}$ is an edge $\mathrm{e}$ and $\mathbf{k}_{\mathrm{e}}$ is ultimately periodic, then $\mathbf{k}_{\mathrm{e}}=l_{\mathrm{e}, 1} v_{\mathrm{e}}^{+\infty}$, with $\left|l_{\mathrm{e}, 1}\right| \geq M$, and $l_{\mathrm{e}, 2}=v_{\mathrm{e}}^{m_{S}}$ for some Lyndon word $v_{\mathrm{e}}$ and $\overline{l_{\mathrm{e}, 2}}=l_{\mathrm{e}, 2}^{\omega+1}=v_{\mathrm{e}}^{\omega+m_{S}}$ is given by (4.3);

$h .3)$ if $\mathrm{g}$ is an edge e, then $r_{\mathrm{e}}=r_{\omega(\mathrm{e})}$ by $(5.3)$, and we let $\widehat{r_{\mathrm{e}}}=\widehat{r_{\omega(\mathrm{e})}}$;

h.4) if $\mathrm{g}$ is a vertex $\mathrm{v}$ and $\mathbf{d}_{\mathrm{v}}$ is non-ultimately periodic, then $r_{\mathrm{v}, 2}$ is the prefix of $r_{\mathrm{v}}$ of length $k$ and $\overline{r_{\mathrm{v}, 2}}$ is given by $(4.2)$;

$h .5)$ if $\mathrm{g}$ is a vertex $\mathrm{v}$ and $\mathbf{d}_{\mathrm{v}}$ is ultimately periodic, then $\mathbf{d}_{\mathrm{v}}=v_{\mathrm{v}}^{-\infty} r_{\mathrm{v}, 1}$ and $r_{\mathrm{v}, 2}=v_{\mathrm{v}}^{m_{S}}$ for some Lyndon word $v_{\mathrm{v}}$ and $\overline{r_{\mathrm{v}, 2}}=r_{\mathrm{v}, 2}^{\omega+1}=v_{\mathrm{v}}^{\omega+m_{S}}$ is given by (4.3).

Finally, for each edge $\mathrm{e} \in \Gamma$, we recall that $c_{\mathrm{e}}=r_{\alpha(\mathrm{e})} l_{\mathrm{e}}$ by (5.3). Hence, we define

$$
\widehat{c_{\mathrm{e}}}=\widehat{r_{\alpha(\mathrm{e})}} \widehat{l_{\mathrm{e}}}
$$

Notice that $\widehat{c_{\mathrm{e}}}$ is a rank $1 \kappa$-term of the form $\bar{x} y \bar{z}=x_{1} x_{2}^{\omega} x_{3} y z_{1} z_{2}^{\omega} z_{3}$, with $x_{1}, x_{3}, y, z_{1}, z_{3} \in A^{*}$ and $x_{2}, z_{2} \in A^{+}$are Lyndon words. Moreover, if the bi-infinite word $\mathbf{w}_{\mathrm{e}}$ is ultimately periodic, then $x_{2}{ }^{-\infty} x_{3} y z_{1} z_{2}{ }^{+\infty}=\mathcal{O}\left(\mathbf{w}_{\mathbf{e}}\right)$.

Definition 5.5 (integers $i_{\mathrm{v}}$ and $i_{\mathrm{e}}$ ) The integers $i_{\mathrm{v}}$ and $i_{\mathrm{e}}$, in (5.2) and (5.3), are chosen sufficiently large so that h.1)-h.5) hold and, for each $\mathrm{g} \in \Gamma$, e $\in \mathcal{E}(\Gamma), \mathrm{f} \in F_{\Gamma}$ and $\mathrm{v}, \mathrm{w} \in \mathcal{V}(\Gamma)$ :

i.1) if $\mathbf{w}_{\mathrm{e}} \sim \mathbf{w}_{\mathrm{f}}$, then $\mathbf{L S l} \models \widehat{c_{\mathrm{e}}}=\widehat{c_{\mathrm{f}}}$;

i.2) if $\mathbf{d}_{\mathrm{v}}=\mathbf{d}_{\mathrm{w}}$, then $r_{\mathrm{v}}=r_{\mathrm{w}}\left(\right.$ whence $\widehat{r_{\mathrm{v}}}=\widehat{r_{\mathrm{w}}}$ by h.4) and h.5)). Notice that if $\mathbf{k}_{\mathrm{v}}=\mathbf{k}_{\mathrm{w}}$, then $l_{\mathrm{v}}=l_{\mathrm{w}}$ by definition $(5.2)$;

i.3) if $\mathbf{w}_{\mathrm{f}}$ is not a bi-infinite factor of $\delta(\mathrm{g})$, then $c_{\mathrm{f}}$ is not a factor of $\delta(\mathrm{g})$; 
i.4) $i_{\mathrm{f}} \geq Q$, where $Q$ is a positive integer in the conditions of Lemma 4.2 with $B$ the set of non-periodic elements of $W_{F}$.

Notice that the integers $i_{\mathrm{v}}$ and $i_{\mathrm{e}}$ may effectively be chosen satisfying these conditions. Indeed, conditions $i .3$ ) and $i .4$ ) are trivially verified as, by definition, we may take $i_{\mathrm{v}}$ and $i_{\mathrm{e}}$ arbitrarily large. To guarantee condition $\left.i .2\right)$ it suffices to let $i_{\mathrm{v}}=i_{\mathrm{w}}$. Now, for $i .1$ ), let $\mathrm{e} \in \mathcal{E}(\Gamma)$ and $\mathrm{f} \in F_{\Gamma}$ be such that $\mathbf{w}_{\mathrm{e}} \sim \mathbf{w}_{\mathrm{f}}$. In particular $\mathbf{d}_{\alpha(\mathrm{e})}$ (resp. $\mathbf{k}_{\mathrm{e}}$ ) is ultimately periodic with period $u$ if and only if $\mathbf{d}_{\alpha(\mathbf{f})}$ (resp. $\mathbf{k}_{\mathbf{f}}$ ) is ultimately periodic with period $u$. So, if we can choose $c_{\mathrm{e}}=c_{\mathrm{f}}$, then clearly $\widehat{c_{\mathrm{e}}}=\widehat{c_{\mathrm{f}}}$, whence $\mathbf{L S l}=\widehat{c_{\mathrm{e}}}=\widehat{c_{\mathrm{f}}}$ trivially. We assume therefore that $c_{\mathrm{e}}=c_{\mathrm{f}}$ is not possible. Then, as one can easily verify, since $l_{\mathrm{e}}$ and $l_{\mathrm{f}}$ are almost freely chosen (in the sense that their only restriction is their length, that must be large enough), $\mathbf{d}_{\alpha(\mathrm{e})}$ and $\mathbf{d}_{\alpha(\mathrm{f})}$ must be ultimately periodic, with period $v=v_{\alpha(\mathrm{e})}=v_{\alpha(\mathrm{f})}$. Moreover, we can make $c_{\mathrm{e}}=v^{m_{S}} u$ and $c_{\mathrm{f}}=v^{m_{S}} u^{\prime}$ for some words $u$ and $u^{\prime}$ such that $u=v^{p} u^{\prime}$ or $u^{\prime}=v^{p} u$. We consider the first case, the other one being symmetric. Then, $\widehat{c_{\mathrm{e}}}=v^{\omega+m_{S}} v^{p} x$ and $\widehat{c_{\mathrm{f}}}=v^{\omega+m_{S}} x$, for some rank $1 \kappa$-term $x$, so that $\mathbf{L S}=\widehat{c_{\mathrm{e}}}=v^{\omega} x=\widehat{c_{\mathrm{f}}}$. This concludes the proof that condition $\left.i .1\right)$ is also satisfiable, thus showing that the integers $i_{\mathrm{v}}$ and $i_{\mathrm{e}}$ fixed in Definition 5.5 are well-defined. We should also notice that $i .1$ ) substitutes condition (a) in [17, Definition 6.4], the analogue of Definition 5.5. This way we correct an error in [17] since that condition (a), which states that "if e and $\mathrm{e}^{\prime}$ are two edges such that $\mathbf{w}_{\mathrm{e}} \sim \mathrm{w}_{\mathrm{e}^{\prime}}$, then $c_{\mathrm{e}}=c_{\mathrm{e}^{\prime}}$ ", is not satisfiable. Indeed, suppose that $\mathrm{e}$ and $\mathrm{e}^{\prime}$ are edges such that $\alpha(\mathrm{e})=\alpha\left(\mathrm{e}^{\prime}\right)=\mathrm{v}, \mathbf{d}_{\mathrm{v}}=a^{-\infty}, \mathbf{k}_{\mathrm{e}}=b a^{+\infty}$ and $\mathbf{k}_{\mathrm{e}^{\prime}}=a b a^{+\infty}$. Then $\mathbf{w}_{\mathrm{e}} \sim \mathbf{w}_{\mathrm{e}^{\prime}}, r_{\mathrm{v}}=a^{i_{\mathrm{v}}}, l_{\mathrm{e}}=b a^{i_{\mathrm{e}}-1}$ and $l_{\mathrm{e}^{\prime}}=a b a^{i_{\mathrm{e}^{\prime}}-2}$, whence $c_{\mathrm{e}}=a^{i_{\mathrm{v}}} b a^{i_{\mathrm{e}}-1} \neq a^{i_{\mathrm{v}}} a b a^{i_{\mathrm{e}^{\prime}}-2}=c_{\mathrm{e}^{\prime}}$.

We end this subsection by fixing integers $N$ and $n$ that will be very important in the rest of the paper. Notice that, for each edge $f \in F_{\Gamma}$ such that $\mathbf{w}_{\mathrm{f}}$ is non-periodic, since the word $c_{\mathrm{f}}$ is a center of $\mathbf{w}_{\mathrm{f}}$ and $i_{\mathrm{f}} \geq Q$ by condition $i .4$ ) above, Lemma 4.2 guarantees the existence of a left-extension $\overleftarrow{c_{\mathrm{f}}}$ of $c_{\mathrm{f}}$ such that: for every $\mathbf{w}_{\mathrm{f}}, \mathbf{w}_{\mathrm{f}^{\prime}} \in B$ and every word $w \in A^{+}$

if two distinct occurrences of $c_{\mathrm{f}}$ and $c_{\mathrm{f}^{\prime}}$ are allowed in $w$ relative to $\overleftarrow{c_{\mathrm{f}}}$ and $\overleftarrow{c_{f^{\prime}}}$ respectively, then these occurrences of $c_{\mathrm{f}}$ and $c_{f^{\prime}}$ are disjoint.

Definition 5.6 (constants $L, n$ and $N$ ) Let $L$ be an integer greater than the lengths of all words $l_{\mathrm{g}}, r_{\mathrm{g}}, c_{\mathrm{e}}$ and $\overleftarrow{c_{\mathrm{e}}}$, and let $n>3 L+k^{\prime \prime}$. Then we fix an integer $N>(2 n+k+1)|A|^{2 n+k}+6 n$.

Notice that $L$ and $n$ were already used in [17]. Notice furthermore that $|A|^{2 n+k}$ is the number of different words of length $2 n+k$ over the alphabet $A$. So, for every word $w \in A^{+}$of length $(2 n+k+1)|A|^{2 n+k}$ there is a word of length $2 n+k$ that has at least two disjoint occurrences in $w$.

\subsection{Application of the $\kappa$-reducibility of $\mathrm{V}$}

We will begin the construction of the $\kappa$-solution $\delta_{*}^{\prime}$ of $\Gamma_{*}$ over $\mathbf{L S l} \vee \mathbf{V}$ by applying the $\kappa$ reducibility of $\mathbf{V}$. However, since we need $\delta_{*}^{\prime}$ to satisfy condition (FT), we will not apply the $\kappa$-reducibility of $\mathbf{V}$ directly to the solution $\delta_{*}$. We first extend the graph $\Gamma_{*}$ and the solution $\delta_{*}$ to a graph $\Gamma_{\bullet}$ and a solution $\delta_{\bullet}$ of an adequate form, and only then we apply the $\kappa$-reducibility of $\mathbf{V}$ (to the solution $\delta_{\bullet}$ ). We will thus obtain a $\kappa$-solution $\delta_{*}^{\prime \prime}$ of $\Gamma_{*}$ over $\mathbf{V}$. Of course, this $\kappa$-solution $\delta_{*}^{\prime \prime}$ is not necessarily a solution over LSl. However, we will show that is possible to 
impose $\delta_{*}^{\prime \prime}$ to be a solution over $\mathbf{L S l}_{P}$ for some large $P$, and then transform $\delta_{*}^{\prime \prime}$ into a $\kappa$-solution $\delta_{*}^{\prime}$ over LSl without losing the property of being a solution over V. For that, we need first to observe some facts about $\delta_{*}$ (see $[10,17]$ for further details) and define a new labeling of the subgraph $\Gamma=\Gamma_{*} \backslash \mathcal{E}_{0}\left(\Gamma_{*}\right)$ of $\Gamma_{*}$ introduced in Subsection 5.2.

Recall that $\varepsilon_{0}\left(\Gamma_{*}\right)$ is the set of edges e $\in \Gamma_{*}$ such that $\delta_{*}(\mathrm{e})$ is a finite word. To simplify the notation, we denote $\mathcal{E}_{0}=\mathcal{E}_{0}\left(\Gamma_{*}\right)$ and let $\mathcal{E}_{\omega}=\mathcal{E}\left(\Gamma_{*}\right) \backslash \mathcal{E}_{0}$. Let $\phi$ be the equivalence relation on $\mathcal{V}\left(\Gamma_{*}\right)$ generated by the relation

$$
\left\{(\mathrm{v}, \mathrm{w}) \in \mathcal{V}\left(\Gamma_{*}\right) \times \mathcal{V}\left(\Gamma_{*}\right): \text { there is an edge } \mathrm{v} \stackrel{\mathrm{e}}{\rightarrow} \mathrm{w} \text { with } \mathrm{e} \in \mathcal{E}_{0}\right\} .
$$

For each vertex $\mathrm{v}$, let $\phi(\mathrm{v})$ be the $\phi$-class of $\mathrm{v}$ and let

$$
\begin{array}{ll}
\mathcal{E}_{\phi(\mathrm{v})}=\left\{\mathrm{e} \in \mathcal{E}_{0}: \omega(\mathrm{e}) \in \phi(\mathrm{v})\right\}=\left\{\mathrm{e} \in \mathcal{E}_{0}: \alpha(\mathrm{e}) \in \phi(\mathrm{v})\right\}, & \mathcal{E}_{\mathrm{v}}=\left\{\mathrm{e} \in \mathcal{E}\left(\Gamma_{*}\right): \omega(\mathrm{e})=\mathrm{v}\right\} \\
\mathcal{E}_{\mathrm{v}, 0}=\mathcal{E}_{\mathrm{v}} \cap \mathcal{E}_{0}, & \mathcal{E}_{\mathrm{v}, \omega}=\mathcal{E}_{\mathrm{v}} \cap \mathcal{E}_{\omega} .
\end{array}
$$

Select a vertex $v_{0}$, called the representative of the class $\phi\left(\mathrm{v}_{0}\right)$, such that $\mathcal{E}_{\phi\left(\mathrm{v}_{0}\right)}$ is non-empty. Let $m_{\phi\left(\mathrm{v}_{0}\right)}$ be the maximum length of labels of non-oriented paths, having no repeated edges, consisting of edges of $\mathcal{E}_{\phi\left(\mathrm{v}_{0}\right)}$. Since $\delta_{*}\left(\mathrm{v}_{0}\right)$ is an infinite pseudoword, there is a factorization

$$
p_{\mathbf{D}} \circ \delta_{*}\left(\mathrm{v}_{0}\right)=z_{\phi\left(\mathrm{v}_{0}\right)} s_{\mathrm{v}_{0}}
$$

where $s_{\mathrm{v}_{0}} \in A^{+}$is a word of length $m_{\phi\left(\mathrm{v}_{0}\right)}$ and $z_{\phi\left(\mathrm{v}_{0}\right)} \in \bar{\Omega}_{A} \mathbf{D}$ is an infinite pseudoword. Let $\mathrm{v} \in \phi\left(\mathrm{v}_{0}\right) \backslash\left\{\mathrm{v}_{0}\right\}$. Select a non-oriented path from $\mathrm{v}_{0}$ to $\mathrm{v}$ consisting of edges of $\varepsilon_{\phi\left(\mathrm{v}_{0}\right)}$ with minimum length of labels. Notice that we may assume, without loss of generality, that $M \geq 2 \max \left\{m_{\phi(\mathrm{v})}: \mathrm{v} \in \mathcal{V}\left(\Gamma_{*}\right), \mathcal{E}_{\phi(\mathrm{v})} \neq \emptyset\right\}$. Let $h=a_{1}^{\epsilon_{1}} \cdots a_{r}^{\epsilon_{r}}$, with $a_{i} \in A$ and $\epsilon_{i} \in\{1,-1\}$, be the label of this path and put $s_{\mathrm{v}}=s_{\mathrm{v}_{0}} h$. By construction, the length of $h$ is at most the length of $s_{\mathrm{v}_{0}}$, that is, $r \leq m_{\phi\left(\mathrm{v}_{0}\right)}$. On the other hand, as $\delta_{*}$ is a solution of $\Gamma_{*}$ over $\mathbf{L S l} \vee \mathbf{V}$, it is also a solution of $\Gamma_{*}$ over $\mathbf{D}$. Therefore, if $\epsilon_{1}=-1$, then $s_{\mathrm{V}_{0}}=w_{1} a_{1}$ whence $s_{\mathrm{V}_{0}} a_{1}^{\epsilon_{1}}=w_{1}$ is a word of length $m_{\phi\left(\mathrm{v}_{0}\right)}-1$. If $\epsilon_{1}=1$, then $s_{\mathrm{v}_{0}} a_{1}^{\epsilon_{1}}=s_{\mathrm{v}_{0}} a_{1}$ is a word of length $m_{\phi\left(\mathrm{v}_{0}\right)}+1$. More generaly, one can verify that for each $i \in\{1, \ldots, r\}, s_{\mathrm{v}_{0}} a_{1}^{\epsilon_{1}} \cdots a_{i}^{\epsilon_{i}}$ is a word of length at least $m_{\phi\left(\mathrm{v}_{0}\right)}-i$. Therefore, $s_{\mathrm{v}}$ belongs to $A^{*}$ and

$$
p_{\mathbf{D}} \circ \delta_{*}(\mathrm{v})=z_{\phi\left(\mathrm{v}_{0}\right)} s_{\mathrm{v}}
$$

Moreover, for every edge $\mathrm{e} \in \mathcal{E}_{\mathrm{v}, \omega}$,

$$
p_{\mathbf{D}} \circ \delta_{*}(\mathrm{e})=p_{\mathbf{D}} \circ \delta_{*}(\mathrm{v})=z_{\phi\left(\mathrm{v}_{0}\right)} s_{\mathrm{v}} .
$$

As a consequence, we deduce that, for every $\mathrm{v} \in \phi\left(\mathrm{v}_{0}\right)$ and $\mathrm{e} \in \mathcal{E}_{\mathrm{v}, \omega}$,

$$
\begin{aligned}
& \delta_{*}(\mathrm{v})=\rho_{\mathrm{v}} \cdot \pi_{\phi\left(\mathrm{v}_{0}\right)} \cdot s_{\mathrm{v}} \\
& \delta_{*}(\mathrm{e})=\rho_{\mathrm{e}} \cdot \pi_{\phi\left(\mathrm{v}_{0}\right)} \cdot s_{\mathrm{v}}
\end{aligned}
$$

for some infinite pseudowords $\pi_{\phi\left(\mathrm{v}_{0}\right)}, \rho_{\mathrm{v}}$ and $\rho_{\mathrm{e}}$. Moreover, if the restriction of $\delta_{*}$ to $\varepsilon_{\phi\left(\mathrm{v}_{0}\right)}$ is not a commuting labeling, then $z_{\phi\left(\mathrm{v}_{0}\right)}=v^{-\infty} u$ for some finite words $u$ and $v \neq 1$, and one may choose $\pi_{\phi\left(\mathrm{v}_{0}\right)}=v^{\omega} u$.

Let us now introduce a new labeling $\delta_{+}$of the subgraph $\Gamma$ which will be useful in the next subsection. This labeling differs from $\delta=\left.\delta_{*}\right|_{\Gamma}$ on the following edges and vertices in which it is defined by: 
- $\delta_{+}(\mathrm{v})=\rho_{\mathrm{v}} \cdot \pi_{\phi\left(\mathrm{v}_{0}\right)}$ for each $\mathrm{v} \in \phi\left(\mathrm{v}_{0}\right)$;

- $\delta_{+}(\mathrm{e})=\rho_{\mathrm{e}} \cdot \pi_{\phi\left(\mathrm{v}_{0}\right)}$ for each edge e such that $\alpha(\mathrm{e}) \notin \phi\left(\mathrm{v}_{0}\right)$ and $\omega(\mathrm{e}) \in \phi\left(\mathrm{v}_{0}\right)$;

- $\delta_{+}(\mathrm{e})=s_{\alpha(\mathrm{e})} \cdot \rho_{\mathrm{e}} \cdot \pi_{\phi\left(\mathrm{v}_{0}\right)}$ for each edge e such that $\alpha(\mathrm{e}) \in \phi\left(\mathrm{v}_{0}\right)$ and $\omega(\mathrm{e}) \in \phi\left(\mathrm{v}_{0}\right)$;

- $\delta_{+}(\mathrm{e})=s_{\alpha(\mathrm{e})} \cdot \delta(\mathrm{e})$ for each edge e such that $\alpha(\mathrm{e}) \in \phi\left(\mathrm{v}_{0}\right)$ and $\omega(\mathrm{e}) \notin \phi\left(\mathrm{v}_{0}\right)$.

We also let $\gamma_{+}: \Gamma \rightarrow S$ be the labeling of $\Gamma$ defined by $\gamma_{+}=\psi \circ \delta_{+}$. In these conditions, by Proposition 3.2, the labeling $\delta_{+}$is a solution of $\Gamma$ over $\mathbf{L S l}$ with respect to $\left(\gamma_{+}, \psi\right)$.

Now, let $P=N+M$ where $N$ is the integer fixed in Definition 5.6. Notice that, by Proposition 3.1, $\bar{\Omega}_{A} \mathbf{L S l} \mathbf{l}_{P}$ is isomorphic to the finite semigroup $A^{+} / \sim_{P}$. For each vertex $\mathrm{v} \in \Gamma_{*}$, consider a factorization

$$
\delta_{*}(\mathrm{v})=u_{\mathrm{v}} \pi_{\mathrm{v}}
$$

where $u_{\mathrm{v}}$ is a finite word of length $M$ (and so $\pi_{\mathrm{v}}$ is an infinite pseudoword). We extend the graph $\Gamma_{*}$ to a graph $\Gamma_{\bullet}$ and extend the solution $\delta_{*}$ of $\Gamma_{*}$ to a solution $\delta_{\bullet}$ of $\Gamma_{\bullet}$ over $\mathbf{L S l} \vee \mathbf{V}$ with respect to a pair $\left(\gamma_{\bullet}, \psi\right)$ as follows. For each vertex $v \in \Gamma$, put a new vertex $z_{v}$ and a new edge $z_{\mathrm{v}} \stackrel{\mathrm{e}_{\mathrm{v}}}{\longrightarrow} \mathrm{v}$ in $\Gamma_{\bullet}$, and let $\delta_{\bullet}\left(\mathrm{z}_{\mathrm{v}}\right)=u_{\mathrm{v}}$ and $\delta_{\bullet}\left(\mathrm{e}_{\mathrm{v}}\right)=\pi_{\mathrm{v}}$. We also let $\gamma_{\bullet}: \Gamma_{\bullet} \rightarrow S$ be the labeling of $\Gamma_{\bullet}$ defined by $\gamma_{\bullet}=\psi \circ \delta_{\bullet}$. Clearly, $\delta_{\bullet}$ is indeed a solution of $\Gamma_{\bullet}$ over $\mathbf{L S I} \vee \mathbf{V}$ with respect to $\left(\gamma_{\bullet}, \psi\right)$.

Since $\delta_{\bullet}$ is a solution of $\Gamma_{\bullet}$ over $\mathbf{V}$, we can apply Lemma 2.1 to the $\kappa$-reducible pseudovariety $\mathbf{V}$ in order to obtain a $\kappa$-solution $\delta_{\bullet}^{\prime}$ over $\mathbf{V}$. Moreover, taking into account Remark 2.2, we can ensure that the prefixes and the suffixes of length $\ell<P$ and the factors of length $\ell \leq P$ of $\delta_{\bullet}(\mathrm{g})$ are preserved for each $\mathrm{g} \in \Gamma_{\bullet}$, because these parameters of $\delta_{\bullet}(\mathrm{g})$ can be tested in the finite semigroup $\bar{\Omega}_{A} \mathbf{L S} \mathbf{S}_{P}$. That is, $\mathbf{L S l}_{P} \models \delta_{\bullet}^{\prime}(\mathrm{g})=\delta_{\bullet}(\mathrm{g})$ for all $\mathrm{g} \in \Gamma_{\bullet}$. As $\delta_{\bullet}$ is a solution over $\mathbf{L S l}_{P}$, it follows that $\delta_{\bullet}^{\prime}$ is also a solution over $\mathbf{L S l}_{P}$. We conclude that $\delta_{\bullet}^{\prime}$ is a $\kappa$-solution of $\Gamma_{\bullet}$ over $\mathbf{L S l}_{P} \vee \mathbf{V}$ with respect to $\left(\gamma_{\bullet}, \psi\right)$ such that,

$$
\forall \mathrm{g} \in \Gamma_{\bullet}, \mathbf{L S l}_{P}=\delta_{\bullet}^{\prime}(\mathrm{g})=\delta_{\bullet}(\mathrm{g}) .
$$

Since $\delta_{\bullet}^{\prime}$ verifies conditions (a) and (b) of Lemma 2.1, we deduce in addition that, for every $\mathrm{g} \in \Gamma_{\bullet}: \delta_{\bullet}(\mathrm{g})$ is an infinite pseudoword if and only if $\delta_{\bullet}^{\prime}(\mathrm{g})$ is an infinite $\kappa$-word; if $\delta_{\bullet}(\mathrm{g})$ is a finite word, then $\delta_{\bullet}^{\prime}(\mathrm{g})=\delta_{\bullet}(\mathrm{g})$. We define a new labeling $\delta_{*}^{\prime \prime}: \Gamma_{*} \rightarrow \bar{\Omega}_{A} \mathbf{S}$ of $\Gamma_{*}$ as follows. For each vertex $\vee \in \mathcal{V}\left(\Gamma_{*}\right)$ and each edge $\mathrm{e} \in \mathcal{E}\left(\Gamma_{*}\right)$, we let

$$
\delta_{*}^{\prime \prime}(\mathrm{v})=\delta_{\bullet}^{\prime}\left(\mathrm{z}_{\mathrm{v}}\right) \delta_{\bullet}^{\prime}\left(\mathrm{e}_{\mathrm{v}}\right)=u_{\mathrm{v}} \delta_{\bullet}^{\prime}\left(\mathrm{e}_{\mathrm{v}}\right) \quad \text { and } \quad \delta_{*}^{\prime \prime}(\mathrm{e})=\delta_{\bullet}^{\prime}(\mathrm{e})
$$

Then $\mathbf{L S l}_{P} \vee \mathbf{V}=\delta_{*}^{\prime \prime}(\mathrm{v})=\delta_{\bullet}^{\prime}(\mathrm{v})$ since $\delta_{\bullet}^{\prime}$ is a solution of $\Gamma_{\bullet}$ over $\mathbf{L S l}_{P} \vee \mathbf{V}$ and $\mathrm{z}_{\mathrm{v}} \stackrel{\mathrm{e}_{\mathrm{v}}}{\longrightarrow} \mathrm{v}$ is an edge of $\Gamma_{\bullet}$. Therefore, the following lemma holds.

Lemma 5.7 The labeling $\delta_{*}^{\prime \prime}$ is a $\kappa$-solution of $\Gamma_{*}$ over $\mathbf{L S l}_{P} \vee \mathbf{V}$ with respect to $\left(\gamma_{*}, \psi\right)$ such that, for each $\mathrm{g} \in \Gamma_{*}$ and each vertex $\mathrm{v} \in \Gamma_{*}$ :

(a) $\delta_{*}(\mathrm{~g})$ is an infinite pseudoword if and only if $\delta_{*}^{\prime \prime}(\mathrm{g})$ is an infinite $\kappa$-word. In particular, $\delta_{*}^{\prime \prime}(\mathrm{v})$ is infinite. Moreover, for each edge $\mathrm{e} \in \mathcal{E}_{0}\left(\Gamma_{*}\right), \delta_{*}^{\prime \prime}(\mathrm{e})=\delta_{*}(\mathrm{e})$;

(b) if $\delta_{*}(\mathrm{v})=u \pi$ where $u \in A^{+}$is a word of length $M$ and $\pi \in \bar{\Omega}_{A} \mathbf{S}$, then $\delta_{*}^{\prime \prime}(\mathrm{v})=u \pi^{\prime \prime}$ where $\pi^{\prime \prime} \in \Omega_{A}^{\kappa} \mathbf{S}$ is such that $\psi(\pi)=\psi\left(\pi^{\prime \prime}\right)$; 
(c) $\mathbf{L S l}_{P}=\delta_{*}^{\prime \prime}(\mathrm{g})=\delta_{*}(\mathrm{~g})$.

Notice that condition (b) above follows immediately from (5.15) and shows that $\delta_{*}^{\prime \prime}$ verifies condition $(\mathrm{FT})$. As we said above, $\delta_{*}^{\prime \prime}$ is not necessarily a solution over LSl. We will use the fact that $\delta_{*}^{\prime \prime}$ coincides with $\delta_{*}$ over $\mathbf{L S} \mathbf{S}_{P}$, to transform it into a $\kappa$-solution $\delta_{*}^{\prime}$ over LSl without losing the property of being a solution over $\mathbf{V}$ that satisfies (FT).

\subsection{Second type $\kappa$-reducibility}

Our next objective is to reduce to the case in which the labels are infinite also on edges and not only on vertices. We follow the technique of the analogous reduction presented in $[17$, Proposition 6.1] for the case of the pseudovariety LSl. Informally speaking, the idea is to eliminate the edges e labeled by finite words and to transfer the suffix $s_{\omega(\mathrm{e})}$ from the label of $\omega(\mathrm{e})$ to the label of all edges beginning in $\omega(\mathrm{e})$. Then, if we are able to determine a solution of the reduced graph that verifies condition (FT) extended to edges, then we can come back and revert the above modifications, thus obtaining the desired $\kappa$-solution of $\Gamma_{*}$.

Consider again the subgraph $\Gamma=\Gamma_{*} \backslash \mathcal{E}_{0}\left(\Gamma_{*}\right)$ of $\Gamma_{*}$ introduced in Subsection 5.2. Let $\mathrm{v} \in \mathcal{V}(\Gamma)$ and $\mathrm{e} \in \mathcal{E}_{\mathrm{v}, \omega}$. By (5.11), (5.12) and Lemma 5.7(c), as $P>M \geq\left|s_{\mathrm{v}}\right|$, we deduce that there exist infinite $\kappa$-words $\tau_{\mathrm{v}}$ and $\tau_{\mathrm{e}}$ such that

$$
\begin{aligned}
& \delta_{*}^{\prime \prime}(\mathrm{v})=\tau_{\mathrm{v}} \cdot s_{\mathrm{v}}, \\
& \delta_{*}^{\prime \prime}(\mathrm{e})=\tau_{\mathrm{e}} \cdot s_{\mathrm{v}} .
\end{aligned}
$$

Define a new labeling $\delta_{0}^{\prime}: \Gamma \rightarrow \Omega_{A}^{\kappa} \mathbf{S}$ of $\Gamma$ as follows. For each vertex $\vee \in \Gamma$ and each edge $\mathrm{e} \in \mathcal{E}_{\mathrm{v}, \omega}$, we let

$\delta_{0}^{\prime}(\mathrm{v})=\left\{\begin{array}{ll}\tau_{\mathrm{v}} & \text { if } \mathcal{E}_{\phi(\mathrm{v})} \neq \emptyset \\ \tau_{\mathrm{v}} \cdot s_{\mathrm{v}} & \text { if } \varepsilon_{\phi(\mathrm{v})}=\emptyset\end{array} \quad\right.$ and $\quad \delta_{0}^{\prime}(\mathrm{e})= \begin{cases}\tau_{\mathrm{e}} & \text { if } \mathcal{E}_{\phi(\alpha(\mathrm{e}))}=\emptyset \text { and } \mathcal{E}_{\phi(\mathrm{v})} \neq \emptyset \\ s_{\alpha(\mathrm{e})} \cdot \tau_{\mathrm{e}} & \text { if } \mathcal{E}_{\phi(\alpha(\mathrm{e}))} \neq \emptyset \text { and } \varepsilon_{\phi(\mathrm{v})} \neq \emptyset \\ \tau_{\mathrm{e}} \cdot s_{\mathrm{v}} & \text { if } \varepsilon_{\phi(\alpha(\mathrm{e}))}=\emptyset \text { and } \mathcal{E}_{\phi(\mathrm{v})}=\emptyset \\ s_{\alpha(\mathrm{e})} \cdot \tau_{\mathrm{e}} \cdot s_{\mathrm{v}} & \text { if } \mathcal{\varepsilon}_{\phi(\alpha(\mathrm{e}))} \neq \emptyset \text { and } \varepsilon_{\phi(\mathrm{v})}=\emptyset\end{cases}$

We also let $\gamma_{0}: \Gamma \rightarrow S$ be the labeling of $\Gamma$ defined by $\gamma_{0}=\psi \circ \delta_{0}^{\prime}$. Since each word $s_{\vee}$ has length lower than $M$ and $N=P-M$, it is straightforward to verify the following.

Lemma 5.8 The labeling $\delta_{0}^{\prime}$ is a $\kappa$-solution of $\Gamma$ over $\mathbf{L S l}_{N}$ with respect to $\left(\gamma_{0}, \psi\right)$ such that $\delta_{0}^{\prime}(\mathrm{g})$ is an infinite $\kappa$-word for each $\mathrm{g} \in \Gamma$. Moreover, if $\mathcal{E}_{0}\left(\Gamma_{*}\right)=\emptyset$, then $\Gamma=\Gamma_{*}, \delta=\delta_{*}$ and $\delta_{0}^{\prime}=\delta_{*}^{\prime \prime}$.

Suppose that we construct a $\kappa$-solution $\delta^{\prime}$ of $\Gamma$ over $\mathbf{L S l}$ with respect to $\left(\gamma_{0}, \psi\right)$ in such a way that, for each $\mathrm{g} \in \Gamma$ and $\mathrm{v}, \mathrm{w} \in \mathcal{V}(\Gamma)$,

(ST.1) if $\delta_{0}^{\prime}(\mathrm{g})=u \pi_{0}^{\prime}$ where $u \in A^{+}$is a word of length $M$ and $\pi_{0}^{\prime} \in \Omega_{A}^{\kappa} \mathbf{S}$, then $\delta^{\prime}(\mathrm{g})=u \pi^{\prime}$ where $\pi^{\prime} \in \Omega_{A}^{\kappa} \mathbf{S}$ is such that $\psi\left(\pi_{0}^{\prime}\right)=\psi\left(\pi^{\prime}\right)$;

(ST.2) if $\mathbf{D} \models \delta(\mathrm{g})=x^{-\infty} y$, where $x \neq 1$ and $y$ are finite words, then $\mathbf{D} \models \delta^{\prime}(\mathrm{g})=\delta_{+}(\mathrm{g})$;

(ST.3) if $\mathbf{L S l}_{N} \models \delta_{0}^{\prime}(\mathrm{v})=\delta_{0}^{\prime}(\mathrm{w})$, then $\mathbf{L S l} \models \delta^{\prime}(\mathrm{v})=\delta^{\prime}(\mathrm{w})$; 
$(\mathrm{ST} .4) \mathbf{V} \models \delta^{\prime}(\mathrm{g})=\delta_{0}^{\prime}(\mathrm{g})$.

If such $\kappa$-solution $\delta^{\prime}$ exists, we say that $\mathbf{L S I} \vee \mathbf{V}$ is ST ("second type") $\kappa$-reducible. We should remark that:

(1) by (ST.1), if e is an edge such that $\mathcal{E}_{\phi(\alpha(\mathrm{e}))} \neq \emptyset$, then $\delta^{\prime}(\mathrm{e})=s_{\alpha(\mathrm{e})} \cdot \pi_{\mathrm{e}}^{\prime}$ for some $\pi_{\mathrm{e}}^{\prime} \in \Omega_{A}^{\kappa} \mathbf{S}$;

(2) conditions h.3) and h.5) of Subsection 5.2 will be fundamental to guarantee that (ST.2) holds. Condition (ST.2) is needed to treat the non-commutative case. For instance, if we have an edge $\mathrm{e} \in \Gamma_{*}$ which is a loop on a vertex $\vee$ and $\delta_{*}(\mathrm{e})$ is a finite word $z$, then $\mathcal{E}(\phi(\mathrm{v})) \neq \emptyset$ and $\delta(\mathrm{v})=\delta_{*}(\mathrm{v})$ may be written in the form $\rho_{\mathrm{v}} v^{\omega} u s_{\mathrm{v}}$, as we saw in (5.11) and the paragraph below it. Notice that $\mathbf{D} \models \delta_{*}(\mathrm{v})=\delta_{*}(\mathrm{v}) z$, whence $\mathbf{D} \models \delta_{*}(\mathrm{v})=v^{\omega} u s_{\mathrm{v}}=z^{\omega}$. Observe that, in this case, $\delta_{+}(\mathrm{v})=\rho_{\mathrm{v}} v^{\omega} u$, whence $\mathbf{D} \models \delta_{+}(\mathrm{v})=v^{\omega} u$. We will define $\delta_{*}^{\prime}(\mathrm{e})$ to be $z$ and $\delta_{*}^{\prime}(\mathrm{v})$ to be $\delta^{\prime}(\mathrm{v}) s_{\mathrm{v}}$. On the other hand, as $\delta_{*}^{\prime}$ will be in particular a solution over $\mathbf{D}$, we will have that $\mathbf{D}=\delta_{*}^{\prime}(\mathrm{v})=\delta_{*}^{\prime}(\mathrm{v}) z$, whence $\mathbf{D} \models \delta_{*}^{\prime}(\mathrm{v})=z^{\omega}$. Therefore, we must have $\mathbf{D} \models \delta^{\prime}(\mathrm{v}) s_{\mathrm{v}}=z^{\omega}=v^{\omega} u s_{\mathrm{v}}$ and, so, $\mathbf{D} \models \delta^{\prime}(\mathrm{v})=v^{\omega} u=\delta_{+}(\mathrm{v})$;

(3) suppose that $\mathrm{v}$ is a vertex such that $\varepsilon_{\phi(\mathrm{v})} \neq \emptyset$ and $\mathrm{v}_{0}$ is the representative of the class $\phi(\mathrm{v})$. Recall that $s_{\mathrm{v}}=s_{\mathrm{v}_{0}} h$ where $h$ is the label of a non-oriented path from $\mathrm{v}_{0}$ to $\mathrm{v}$ consisting of edges of $\mathcal{E}_{\phi\left(\mathrm{v}_{0}\right)}$. Then LSl $\models \delta_{*}(\mathrm{v})=\delta_{*}\left(\mathrm{v}_{0}\right) h$, whence $\mathbf{L S l}_{P} \models \delta_{*}^{\prime \prime}(\mathrm{v})=\delta_{*}^{\prime \prime}\left(\mathrm{v}_{0}\right) h$ by Lemma 5.7(c). Since $\left|s_{\mathrm{v}}\right| \leq M$ and $P=N+M$ it follows from the definition of $\delta_{0}^{\prime}$ that $\mathbf{L S l}_{N} \models \delta_{0}^{\prime}(\mathrm{v})=\delta_{0}^{\prime}\left(\mathrm{v}_{0}\right)$. Therefore, by (ST.3), LSl $\models \delta^{\prime}(\mathrm{v})=\delta^{\prime}\left(\mathrm{v}_{0}\right)$;

(4) in case $\varepsilon_{0} \neq \emptyset$, the property of being a solution over $\mathbf{V}$ may have been lost in the passage from the labeling $\delta_{*}^{\prime \prime}$ to $\delta_{0}^{\prime}$. However, as a consequence of condition (ST.4), we will recover that property in the passage from $\delta^{\prime}$ to $\delta_{*}^{\prime}$ since in that passage we will revert the transformations made from $\delta_{*}^{\prime \prime}$ to $\delta_{0}^{\prime}$.

We define $\delta_{*}^{\prime}$ to be the labeling of $\Gamma_{*}$ such that:

- for each vertex $\vee \in \Gamma_{*}$,

$$
\delta_{*}^{\prime}(\mathrm{v})= \begin{cases}\delta^{\prime}(\mathrm{v}) \cdot s_{\mathrm{v}} & \text { if } \mathcal{E}_{\phi(\mathrm{v})} \neq \emptyset \\ \delta^{\prime}(\mathrm{v}) & \text { if } \mathcal{\varepsilon}_{\phi(\mathrm{v})}=\emptyset\end{cases}
$$

- for each edge e $\in \mathcal{E}_{0}, \delta_{*}^{\prime}(\mathrm{e})=\delta_{*}(\mathrm{e})$;

- for each edge $\mathrm{e} \in \mathcal{E}_{\mathrm{v}, \omega}$,

$$
\delta_{*}^{\prime}(\mathrm{e})= \begin{cases}\delta^{\prime}(\mathrm{e}) \cdot s_{\mathrm{v}} & \text { if } \mathcal{E}_{\phi(\alpha(\mathrm{e}))}=\emptyset \text { and } \mathcal{E}_{\phi(\mathrm{v})} \neq \emptyset \\ \pi_{\mathrm{e}}^{\prime} \cdot s_{\mathrm{v}} & \text { if } \mathcal{E}_{\phi(\alpha(\mathrm{e}))} \neq \emptyset \text { and } \mathcal{E}_{\phi(\mathrm{v})} \neq \emptyset \\ \delta^{\prime}(\mathrm{e}) & \text { if } \mathcal{E}_{\phi(\alpha(\mathrm{e}))}=\emptyset \text { and } \mathcal{E}_{\phi(\mathrm{v})}=\emptyset \\ \pi_{\mathrm{e}}^{\prime} & \text { if } \mathcal{E}_{\phi(\alpha(\mathrm{e}))} \neq \emptyset \text { and } \mathcal{E}_{\phi(\mathrm{v})}=\emptyset\end{cases}
$$

Then, as one can easily verify, $\delta_{*}^{\prime}$ is indeed a $\kappa$-solution of $\Gamma_{*}$ over $\mathbf{L S I} \vee \mathbf{V}$ with respect to $\left(\gamma_{*}, \psi\right)$ which satisfies condition (FT). This proves the following result.

Proposition 5.9 If $\mathbf{L S l} \vee \mathbf{V}$ is $S T \kappa$-reducible, then it is $\kappa$-reducible. 
The objective of the rest of the paper is to prove the ST $\kappa$-reducibility of $\mathbf{L S I} \vee \mathbf{V}$. We assume therefore that $M$ and $N$ are positive integers (with $N$ given by Definition 5.6) and that $\delta_{0}^{\prime}$ is a $\kappa$-solution of a finite graph $\Gamma$ over $\mathbf{L} \mathbf{S l}_{N}$ with respect to a pair $\left(\gamma_{0}, \psi\right)$, labeling each element of $\Gamma$ by an infinite $\kappa$-word. We need to construct a $\kappa$-solution $\delta^{\prime}$ of $\Gamma$ over LSl with respect to $\left(\gamma_{0}, \psi\right)$ satisfying conditions (ST.1)-(ST.4).

Just to allow us to use the notation relative to $\delta$ already introduced in Subsection 5.2, we will assume furthermore that $\varepsilon_{0}\left(\Gamma_{*}\right)=\emptyset$ so that $\Gamma=\Gamma_{*}, \delta=\delta_{*}=\delta_{+}, \delta_{0}^{\prime}=\delta_{*}^{\prime \prime}$ and $\gamma=\gamma_{*}=\gamma_{+}=\gamma_{0}$. Notice that, by Lemma 5.7(c), $\mathbf{L S l}_{P}=\delta_{*}^{\prime \prime}(\mathrm{g})=\delta_{*}(\mathrm{~g})$ for every $\mathrm{g} \in \Gamma_{*}$. Therefore, that assumption is harmless since it serves merely to avoid having to rearrange the notation in order to handle the transfer of the suffix $s_{\omega(\mathrm{e})}$ from the label of some vertices $\omega(\mathrm{e})$ to the label of all edges beginning in $\omega(\mathrm{e})$. So, with this new notation and for latter reference, we have that

$$
\forall \mathrm{g} \in \Gamma, \mathbf{L S l}_{N} \models \delta_{0}^{\prime}(\mathrm{g})=\delta(\mathrm{g})
$$

\section{Transformation algorithm}

We begin the description of the algorithm of transformation of $\delta_{0}^{\prime}$ into the $\kappa$-solution $\delta^{\prime}$ by explaining some details of the process. For each $\mathrm{g} \in \Gamma$, we fix a $\kappa$-term $w_{\mathrm{g}, 0} \in T_{A}^{\kappa}$ such that $\varepsilon\left(w_{\mathrm{g}, 0}\right)=\delta_{0}^{\prime}(\mathrm{g})$. We will apply to each of these $\kappa$-terms $w_{\mathrm{g}, 0}$ a process of transformation, consisting of 5 steps, described in the following subsections. The $\kappa$-term which arises after the $j$ th step will be denoted by $w_{\mathrm{g}, j}$ and we let $\delta_{j}^{\prime}(\mathrm{g})=\varepsilon\left(w_{\mathrm{g}, j}\right)$. So, for each $j \in\{0, \ldots, 5\}$,

$$
\begin{aligned}
\delta_{j}^{\prime}: \Gamma & \longrightarrow \Omega_{A}^{\kappa} \mathbf{S} \\
\mathbf{g} & \longmapsto \varepsilon\left(w_{\mathrm{g}, j}\right)
\end{aligned}
$$

defines a labeling of $\Gamma$ by $\kappa$-words. The $\kappa$-solution $\delta^{\prime}$ over LSl will then be defined as $\delta^{\prime}=\delta_{5}^{\prime}$. So, the initial labeling $\delta_{0}^{\prime}$, which is a solution over $\mathbf{L} \mathbf{S l}_{N}$, may be seen as a sort of "initial approximation" of the solution $\delta^{\prime}$ and the objective of our algorithm is to determine a sequence $\delta_{1}^{\prime}, \ldots, \delta_{5}^{\prime}$ of successive refinements of $\delta_{0}^{\prime}$, ending up with the desired solution $\delta^{\prime}$. The last step of the process of transformation will closely follow some parts of the one described by the first author and Teixeira in [17] to prove the $\kappa$-reducibility of LSl, which will make our work easier. Apart having to preserve the value over $\mathbf{V}$ (condition (ST.4)), which is easy, there is however a major difference which makes the algorithm more complex: the initial $\kappa$-terms $w_{\mathrm{g}, 0}$ are infinite, while in [17] they were finite. So, while for LSl only one transformation rule was used, for the process of transformation in the $\mathbf{L S I} \vee \mathbf{V}$ case we need to use several rewriting rules for $\kappa$-terms, which we proceed to describe.

\subsection{Rewriting rules}

We will use the following set $\Sigma$ of rewriting rules for $\kappa$-terms (where $x, y \in T_{A}^{\kappa}$ and $i, j \in \mathbb{Z}$ )

(R.1) $\left(x^{\omega+i}\right)^{\omega+j} \rightarrow x^{\omega+i j}$,

(R.2) $\left(x^{i}\right)^{\omega+j} \leftrightarrow x^{\omega+i j}, \quad(i>0)$

(R.3) $x^{\omega+i} x^{\omega+j} \leftrightarrow x^{\omega+i+j}$, 
(R.4) $x^{i} x^{\omega+j} \leftrightarrow x^{\omega+i+j}, x^{\omega+j} x^{i} \leftrightarrow x^{\omega+i+j}, \quad(i>0)$

(R.5) $(x y)^{\omega+i} \rightarrow x(y x)^{\omega+i-1} y$,

(R.6) $u \rightarrow \bar{u}, \quad\left(u \in A^{+}\right.$with $\bar{u}$ defined in (4.2) and (4.3)).

As one notices, rules (R.1)-(R.5) come from the identities (3.1)-(3.5) above, which hold in $\mathbf{S}$. On the contrary, rule (R.6) does not preserve the value of the $\kappa$-term over $\mathbf{S}$, but it preserves the value over $\mathbf{V}$ as referred in Subsection 4.2. We also observe that, as in rules (R.2)-(R.4), we could also permit, in rules (R.1), (R.5) and (R.6), the transformations from the terms on the right to the ones on the left. Since we don't need to use those transformations, we don't include them in the rules. If we start with a $\kappa$-term that contains a subterm that looks like the left-hand side of rule (R.m) and rewrite this subterm to look like the right-hand side, then this application of rule (R.m) will be called a left-right transformation of type $m$. The reverse transformation will be called a right-left transformation of type $m$. For transformations of type 4 , we will distinguish between $4_{L}$ and $4_{R}$ depending on whether $x^{i} x^{\omega+j} \leftrightarrow x^{\omega+i+j}$ or $x^{\omega+j} x^{i} \leftrightarrow x^{\omega+i+j}$ is used.

As mentioned above, starting from each $w_{\mathrm{g}, 0}$, we will use $\Sigma$ to successively derive $\kappa$-terms $w_{\mathrm{g}, 1}, \ldots, w_{\mathrm{g}, 5}$ and define $\delta_{j}^{\prime}(\mathrm{g})=\varepsilon\left(w_{\mathrm{g}, j}\right)$, for $j \in\{1, \ldots, 5\}$, and $\delta^{\prime}=\delta_{5}^{\prime}$. Since each rule of $\Sigma$ preserves the value over the fixed semigroup $S$, we will have $\psi\left(w_{\mathrm{g}, 0}\right)=\psi\left(w_{\mathrm{g}, j}\right)$. So, as $\psi \circ \delta_{0}^{\prime}=\gamma_{0}$, this equality guarantees that $\psi \circ \delta_{j}^{\prime}=\gamma_{0}$ and, in particular, that $\psi \circ \delta^{\prime}=\gamma_{0}$. Notice that we want $\delta_{5}^{\prime}$ to be a $\kappa$-solution over LSl. As $\delta_{0}^{\prime}$ is a solution over $\mathbf{L S l}_{N}$ and, so, not necessarily a solution over LSl (in that case we would have nothing to do), it is rule (R.6) that will allow us to convert $\delta_{0}^{\prime}$ into a solution $\delta_{5}^{\prime}$ over LSI. Indeed, this rule is the only one that changes the value of $\kappa$-terms over $\mathbf{S}$ (and over $\mathbf{L S l}$ ). The choice of a large enough $N$ was made so that conversion could be possible. Furthermore, since each rule of $\Sigma$ preserves the value over $\mathbf{V}$, condition (ST.4) will hold trivially.

\subsection{Initial steps}

We begin by noticing that the $\kappa$-term $w_{\mathrm{g}, 0}$ may be chosen already in the form $w_{\mathrm{g}, 0}=l_{\mathrm{g}} w_{\mathrm{g}, 0}^{\prime} r_{\mathrm{g}}$, where $l_{\mathrm{g}}$ and $r_{\mathrm{g}}$ are the finite words defined in (5.2)-(5.3). Indeed, $l_{\mathrm{g}}$ and $r_{\mathrm{g}}$ have length at most $L$ and, so, smaller than $N$ by Definition 5.6. Therefore, by (5.18), $l_{\mathrm{g}}$ and $r_{\mathrm{g}}$ are, respectively, a prefix and a suffix of the $\kappa$-word $\delta_{0}^{\prime}(\mathrm{g})$. So, given any $\kappa$-term $w$ such that $\varepsilon(w)=\delta_{0}^{\prime}(\mathrm{g})$, to make appear $l_{\mathrm{g}}$ and $r_{\mathrm{g}}$ it would suffice, if necessary, to apply right-left transformations of type 4 , to obtain the $\kappa$-term $w_{\mathrm{g}, 0}$ of the desired form. The main objective of this choice of $w_{\mathrm{g}, 0}$ is explained in the following statement.

Remark 6.1 The next steps will not change the prefix $l_{\mathrm{g}}$ nor the suffix $r_{\mathrm{g}}$ of $w_{\mathrm{g}, 0}$, until step 5 where they will be replaced respectively by $\widehat{l_{\mathrm{g}}}=l_{\mathrm{g}, 1} \overline{l_{\mathrm{g}, 2}}$ and $\widehat{r_{\mathrm{g}}}=\overline{r_{\mathrm{g}, 2}} r_{\mathrm{g}, 1}$, the rank $1 \kappa$-terms defined in (5.7).

As a consequence, we may conclude in advance that the labeling $\delta^{\prime}=\delta_{5}^{\prime}$ will be a $\kappa$-solution over LI. To justify this statement let us show that the labeling $\delta_{5}^{\prime}$ is such that $\mathbf{L I}$ satisfies each pseudoidentity $\delta_{5}^{\prime}(\alpha(\mathrm{e})) \delta_{5}^{\prime}(\mathrm{e})=\delta_{5}^{\prime}(\omega(\mathrm{e}))$. By condition h.3) of Subsection 5.2, $\widehat{\mathrm{r}_{\mathrm{e}}}=\widehat{r_{\omega(\mathrm{e})}}$, whence the pseudoidentity will hold over $\mathbf{D}$. On the other hand, by (5.1), $\mathbf{k}_{\alpha(\mathrm{e})}=\mathbf{k}_{\omega(\mathrm{e})}$ since 
$\delta$ is a solution of $\Gamma$ over $\mathbf{K}$. Therefore $l_{\alpha(\mathrm{e})}=l_{\omega(\mathrm{e})}$ by $(5.2)$, and $\widehat{l_{\alpha(\mathrm{e})}}=\widehat{l_{\omega(\mathrm{e})}}$ by $\left.h .1\right)$, whence the pseudoidentity will be valid over $\mathbf{K}$.

Moreover, as $\widehat{l_{\mathrm{g}}}=l_{\mathrm{g}, 1} \overline{l_{\mathrm{g}, 2}}$ and $l_{\mathrm{g}, 1}$ is by definition a word of length at least $M$, condition (ST.1) will be verified. On the other hand, as $\widehat{r_{\mathrm{g}}}=\bar{r}_{\mathrm{g}, 2} r_{\mathrm{g}, 1}$ and we are assuming $\delta=\delta_{+}$, (ST.2) is a consequence of conditions h.3) and h.5). Therefore, since condition (ST.4) is also guaranteed in advance, it suffices to assure that $\delta_{5}^{\prime}$ will verify (ST.3).

We say that a word $u \in A^{+}$is a basis of a $\kappa$-term $w$ if $w$ has a subterm of the form $u^{\alpha}$ where $\alpha \in \omega+\mathbb{Z}$. For instance, the bases of the $\kappa$-term in (3.6) are $b a, a^{3}$ and $a^{2}$.

Step 1. We describe the first step of the algorithm which will transform each $\kappa$-term $w_{\mathrm{g}, 0}$ into a $\kappa$-term $w_{\mathrm{g}, 1}$, with the following properties:

s1.1) all bases of $w_{\mathrm{g}, 1}$ are Lyndon words with length at most $k$;

s1.2) every factor of length $N$ of the $\kappa$-word $\varepsilon\left(w_{\mathrm{g}, 0}\right)$ is also a factor of the $\kappa$-word $\varepsilon\left(w_{\mathrm{g}, 1}\right)$;

s1.3) $w_{\mathrm{g}, 1}$ is of the form $w_{\mathrm{g}, 1}=l_{\mathrm{g}} w_{\mathrm{g}, 1}^{\prime} r_{\mathrm{g}}$.

Although it would not be necessary, we can assume that $w_{\mathrm{g}, 0}$ has no subterms of the form $\left(x^{\omega+i}\right)^{\omega+j}$ since, otherwise, we could apply to it all possible transformations of type 1 to obtain a $\kappa$-term with that property. Consider each occurrence in $w_{\mathrm{g}, 0}$ of a subterm of the form $u^{\omega+p}$ with $u \in A^{+}$and $p \in \mathbb{Z}$. Let $\ell_{u}$ be a positive integer such that $u^{\ell_{u}}$ has all the factors of length $N$ of the bi-infinite word $u^{\infty}$. Let also $p^{\prime}$ be an integer such that $\ell_{u} p^{\prime}+p \geq \ell_{u}$ and fix a factorization $u^{\ell_{u}}=u_{1} u_{2} u_{3}$ with $\left|u_{1}\right| \geq N,\left|u_{2}\right|=k$ and $\left|u_{3}\right| \geq N$. We transform the occurrence $u^{\omega+p}$ as follows, applying successively transformations of types $4_{R}, 2$ and 6 ,

$$
u^{\omega+p} \rightarrow u^{\omega-\ell_{u} p^{\prime}} u^{\ell_{u} p^{\prime}+p} \rightarrow\left(u^{\ell_{u}}\right)^{\omega-p^{\prime}} u^{\ell_{u} p^{\prime}+p} \rightarrow\left(u_{1} \overline{u_{2}} u_{3}\right)^{\omega-p^{\prime}} u^{\ell_{u} p^{\prime}+p} .
$$

Denote by $w_{\mathrm{g}, 1}$ the $\kappa$-term obtained after all these transformations. Recall that, by (4.2), $\overline{u_{2}}$ is of the form $\overline{u_{2}}=a_{1} \cdots a_{i^{\prime}-1}\left(a_{i^{\prime}} \cdots a_{j} a_{i} \cdots a_{i^{\prime}-1}\right)^{\omega} a_{i^{\prime}} \cdots a_{k}$. So, with the above modifications, each basis $u$ of $w_{\mathrm{g}, 0}$ was transformed into a basis $a_{i^{\prime}} \cdots a_{j} a_{i} \cdots a_{i^{\prime}-1}$ of $w_{\mathrm{g}, 1}$ of length at most $k$. Since $a_{i^{\prime}} \cdots a_{j} a_{i} \cdots a_{i^{\prime}-1}$ is a Lyndon word, this shows property s1.1). Moreover, $u^{\ell_{u} p^{\prime}+p}$ has all the factors of length $N$ of $u^{\infty}$ because $u^{\ell_{u}}$ has that property and $\ell_{u} p^{\prime}+p \geq \ell_{u}$. So, as $\left|u_{1}\right|,\left|u_{3}\right| \geq N$, property s1.2) also holds. Notice that the converse of s1.2) may not hold because the use of rule (R.6) may have introduced new factors of length $N$ on the $\kappa$-word $\varepsilon\left(w_{\mathrm{g}, 1}\right)$. Hence, contrary to what happens with $\delta_{0}^{\prime}$, the labeling $\delta_{1}^{\prime}$ may not be a solution over $\mathbf{L S l}_{N}$. Finally, property s1.3) holds since $w_{\mathrm{g}, 0}=l_{\mathrm{g}} w_{\mathrm{g}, 0}^{\prime} r_{\mathrm{g}}$ and the above transformations did not modify the prefix $l_{\mathrm{g}}$ nor the suffix $r_{\mathrm{g}}$.

Notice that for the purpose of this step, the factorization $u^{\ell_{u}}=u_{1} u_{2} u_{3}$ which determines the occurrence of $u_{2}$ where rule (R.6) is applied could be chosen arbitrarily. However, Step 3 below will need the factorization to verify some conditions that we proceed to describe. First, we make a list $z_{1}, z_{2}, \ldots, z_{r}$ enumerating the powers $u^{\ell_{u}}$ for all bases $u$ of all $\kappa$-terms $w_{\mathrm{g}, 0}$. For each $q \in\{1, \ldots, r\}$, we choose a factor $z_{q}^{\prime}=u_{q, 1} u_{q, 2} u_{q, 3}$ of $z_{q}$, with $\left|u_{q, 1}\right|=\left|u_{q, 3}\right|=n$ and $\left|u_{q, 2}\right|=k$, where $n$ is the positive integer fixed in Definition 5.6. Moreover, for $q \in\{2, \ldots, r\}$,

if $q$ is such that $z_{m}^{\prime}$ is a factor of $z_{q}$ for some $m<q$, then we let $z_{q}^{\prime}=z_{m}^{\prime}$. 
We fix an occurrence of $z_{q}^{\prime}=u_{q, 1} u_{q, 2} u_{q, 3}$ in $z_{q}$ and the middle occurrence $u_{q, 2}$ is where rule (R.6) is applied.

Before proceeding with the next step, we introduce some terminology. Let $w$ be a $\kappa$ term and suppose that $w$ can be factorized as $w=w_{1} w^{\prime} w_{2}$. If $w^{\prime}=u_{1}^{\alpha_{1}} u^{\prime} u_{2}^{\alpha_{2}}$ is a rank $1 \kappa$-term in reduced form, then $w^{\prime}$ is called a crucial factor of $w$. If $w_{1}=1$ and $w^{\prime}=$ $u^{\prime} u^{\alpha}$ is a rank $1 \kappa$-term in reduced form, then $w^{\prime}$ is called a crucial prefix of $w$. Notice that $w$ has at most one crucial prefix. The definition of a crucial suffix of $w$ is dual. For instance, the $\kappa$-term $w=a(a b)^{\omega-3} b^{4}\left(b a^{\omega-2}\right)^{\omega+1} a$ has crucial prefix $a(a b)^{\omega-3}$, has no crucial factors and has no crucial suffix. Now, using the rewriting rules, we could transform $w$ into $w_{1}=a(a b)^{\omega-3} b^{5} a^{\omega-2} b a^{\omega-2}\left(b a^{\omega-2}\right)^{\omega-3} b a^{\omega-2} b a^{\omega-2} a$. The $\kappa$-term $w_{1}$ has crucial prefix $a(a b)^{\omega-3}$, crucial suffix $a^{\omega-2} a$ and crucial factors $(a b)^{\omega-3} b^{5} a^{\omega-2}$ and $a^{\omega-2} b a^{\omega-2}$. Notice that $\varepsilon_{A, \mathbf{K}}^{\kappa}(w)=a(a b)^{+\infty}, \varepsilon_{A, \mathbf{D}}^{\kappa}(w)=a^{-\infty}$ and $B_{w}=\left\{(a b)^{-\infty} b^{5} a^{+\infty}, a^{-\infty} b a^{+\infty}\right\}$.

Step 2. Consider the $\kappa$-term $w_{\mathrm{g}, 1}$. We show it is possible to apply only right-left transformations of type 4 to $w_{\mathrm{g}, 1}$ to derive a $\kappa$-term $w_{\mathrm{g}, 2}$ such that:

s2.1) if $\varepsilon_{A, \mathbf{K}}^{\kappa}\left(w_{\mathrm{g}, 1}\right)=u^{\prime} u^{+\infty}$, then $w_{\mathrm{g}, 2}$ has a crucial prefix of the form $u^{\prime} u^{\alpha}$;

$\mathrm{s} 2.2)$ if $\varepsilon_{A, \mathbf{D}}^{\kappa}\left(w_{\mathrm{g}, 1}\right)=u^{-\infty} u^{\prime}$, then $w_{\mathrm{g}, 2}$ has a crucial suffix of the form $u^{\alpha} u^{\prime}$;

s2.3) $w_{\mathrm{g}, 2}$ has a crucial factor of the form $u_{1}^{\alpha_{1}} u^{\prime} u_{2}^{\alpha_{2}}$ if and only if $u_{1}^{-\infty} u^{\prime} u_{2}^{+\infty} \in B_{w_{\mathrm{g}, 1}}$;

s2.4) $w_{\mathrm{g}, 2}$ is of the form $w_{\mathrm{g}, 2}=l_{\mathrm{g}} w_{\mathrm{g}, 2}^{\prime} r_{\mathrm{g}}$;

s2.5) the $\kappa$-term $w_{\mathrm{g}, 2}$ and the $\kappa$-words $\varepsilon\left(w_{\mathrm{g}, 1}\right)$ and $\varepsilon\left(w_{\mathrm{g}, 2}\right)$ have the same finite factors of length $N$.

To transform the $\kappa$-term $w_{\mathrm{g}, 1}$ into a rank $1 \kappa$-term $z_{1}$ such that $\mathbf{L S l} \models w_{\mathrm{g}, 1}=z_{1}$, it would suffice, by Lemma 3.3, to apply all possible transformations $x^{\omega+i} \rightarrow x^{2}$ with $x$ an infinite $\kappa$-term. Since the bases of $w_{\mathrm{g}, 1}$ (and so of $z_{1}$ too) are already Lyndon words, to transform $z_{1}$ into a $\kappa$-term $z_{2}$ in reduced form it would then suffice to apply left-right transformations of types 4 and 3 to replace all possible subterms of the form $u^{\omega+i} u^{\ell} u^{\omega+j}$ by $u^{\omega+i+\ell+j}$. On the other hand, $B_{w_{\mathrm{g}, 1}}=B_{z_{2}}$ by Proposition 3.2. So, as one can convince oneself easily, it is possible to obtain from $w_{\mathrm{g}, 1}$ a $\kappa$-term $w_{\mathrm{g}, 1}(1)$, using right-left transformations of type 4 of the form $x^{\omega+i} \rightarrow x^{\omega+i-2} x^{2}$ and $x^{\omega+i} \rightarrow x^{2} x^{\omega+i-2}$ with $x$ infinite, such that $w_{\mathrm{g}, 1}(1)$ verifies s2.1)s2.3). Notice that we were not interested in defining a unique form to represent a bi-infinite word $u_{1}^{-\infty} u^{\prime} u_{2}^{+\infty}$, whence this word can also be represented for instance by $u_{1}^{-\infty} u_{1}^{i} u^{\prime} u_{2}^{j} u_{2}^{+\infty}$ for every non-negative integers $i$ and $j$. So, for a given non-periodic bi-infinite factor $\mathbf{w}^{\prime}$ of $w_{\mathrm{g}, 1}$, what $\left.\mathrm{s} 2.3\right)$ says is that $w_{\mathrm{g}, 2}$ contains a crucial factor of the form $u_{1}^{\alpha_{1}} u^{\prime} u_{2}^{\alpha_{2}}$ for some representation $u_{1}^{-\infty} u^{\prime} u_{2}^{+\infty}$ of $\mathbf{w}^{\prime}$. That $w_{\mathrm{g}, 1}(1)$ verifies $\left.\mathrm{s} 2.4\right)$ follows from s1.3) and the fact that the above transformations do not modify the prefix $l_{\mathrm{g}}$ nor the suffix $r_{\mathrm{g}}$ of $w_{\mathrm{g}, 1}$.

Notice that, since $w_{\mathrm{g}, 1}(1)$ verifies properties s2.1)-s2.3), $\varepsilon_{A, \mathbf{K}}^{\kappa}\left(w_{\mathrm{g}, 1}(1)\right)=\varepsilon_{A, \mathbf{K}}^{\kappa}\left(w_{\mathrm{g}, 1}\right)$, $\varepsilon_{A, \mathbf{D}}^{\kappa}\left(w_{\mathrm{g}, 1}(1)\right)=\varepsilon_{A, \mathbf{D}}^{\kappa}\left(w_{\mathrm{g}, 1}\right)$ and $B_{w_{\mathrm{g}, 1}(1)}=B_{w_{\mathrm{g}, 1}}$. Let $v \in A^{+}$be a word of length $N$. If $v$ is a factor of the $\kappa$-term $w_{\mathrm{g}, 1}(1)$ (i.e., if $w_{\mathrm{g}, 1}(1)$ can be factorized as $w_{\mathrm{g}, 1}(1)=w_{1} v w_{2}$ ), then $v$ is trivially a factor of the $\kappa$-word $\varepsilon\left(w_{\mathrm{g}, 1}(1)\right)$. Reciprocally, suppose that $v$ is a factor of the $\kappa$-word $\varepsilon\left(w_{\mathrm{g}, 1}(1)\right)$. Then $v$ is either: 1$)$ a factor of $\left.\varepsilon_{A, \mathbf{K}}^{\kappa}\left(w_{\mathrm{g}, 1}(1)\right) ; 2\right)$ a factor of $\varepsilon_{A, \mathbf{D}}^{\kappa}\left(w_{\mathrm{g}, 1}(1)\right)$; 
3) a factor of a bi-infinite factor $u_{1}^{-\infty} u^{\prime} u_{2}^{+\infty} \in B_{w_{\mathrm{g}, 1}(1)}$. In case 3$), w_{\mathrm{g}, 1}(1)$ has a crucial factor of the form $u_{1}^{\alpha_{1}} u^{\prime} u_{2}^{\alpha_{2}}$ by s2.3). Then, applying right-left transformations of type 4 if necessary, we could substitute the factor $u_{1}^{\alpha_{1}} u^{\prime} u_{2}^{\alpha_{2}}$ by $u_{1}^{\alpha_{1}-i} u_{1}^{i} u^{\prime} u_{2}^{i} u_{2}^{\alpha_{2}-i}$ for a sufficiently large positive integer $i$ such that $v$ is a factor of $u_{1}^{i} u^{\prime} u_{2}^{i}$, whence $v$ would be a factor of the $\kappa$-term thus obtained from $w_{\mathrm{g}, 1}(1)$. The cases 1 ) and 2) are treated analogously. Proceeding like this for all words $v$ of length $N$, we obtain a $\kappa$-term $w_{\mathrm{g}, 2}$ with the same finite factors of length $N$ of the $\kappa$-word $\varepsilon\left(w_{\mathrm{g}, 2}\right)$. On the other hand, since rule (R.6) was not applied in this step, the $\kappa$-words $\varepsilon\left(w_{\mathrm{g}, 1}\right)$ and $\varepsilon\left(w_{\mathrm{g}, 2}\right)$ have the same finite factors of length $N$. So, $w_{\mathrm{g}, 2}$ verifies s2.1)-s2.5).

\subsection{Construction of the same bases}

Recall that we want to construct $\delta^{\prime}=\delta_{5}^{\prime}$ to be a $\kappa$-solution over LSl. By Remark 6.1, if we preserve some simple parameters, we will get a solution over LI. So, in view of Proposition 3.4, it suffices in addition to build the $\kappa$-terms $w_{\mathrm{g}, 5}$ in such a way that, for each edge $\mathrm{e} \in \Gamma$, the $\kappa$-terms $w_{\alpha(\mathrm{e}), 5} w_{\mathrm{e}, 5}$ and $w_{\omega(\mathrm{e}), 5}$ have the same non-periodic bi-infinite factors, that is, $B_{w_{\alpha(\mathrm{e}), 5} w_{\mathrm{e}, 5}}=B_{w_{\omega(\mathrm{e}), 5}}$. As one may easily verify, a necessary condition for that to happen is that, for each edge e $\in \Gamma$, the $\kappa$-terms $w_{\alpha(\mathrm{e}), 5} w_{\mathrm{e}, 5}$ and $w_{\omega(\mathrm{e}), 5}$ have the same bases. Moreover, since we need condition (ST.3) to hold, it is also necessary that, if $\mathrm{v}, \mathrm{w} \in \mathcal{V}(\Gamma)$ are vertices such that $\mathbf{L S l}_{N}=w_{\mathrm{v}, 0}=w_{\mathrm{w}, 0}$, then the $\kappa$-terms $w_{\mathrm{v}, 5}$ and $w_{\mathrm{w}, 5}$ have the same bases. With the purpose of assuring these properties, we will transform the bases of each $\kappa$-term $w_{\mathrm{g}, 2}$. Since the $\kappa$-terms $w_{\alpha(\mathrm{e}), 5} w_{\mathrm{e}, 5}$ and $w_{\omega(\mathrm{e}), 5}$ involve three elements of $\Gamma$ (namely e, $\alpha(\mathrm{e})$ and $\omega(\mathrm{e})$ ), we cannot perform the transformations in each $\mathrm{g} \in \Gamma$ independently from the other elements $\mathrm{g}^{\prime}$ of $\Gamma$; each element of $\Gamma$ interferes with all the elements of its connected component (this is, of course, the reason of the difficulty in proving the reducibility of pseudovarieties in general).

Step 3. In this step we construct $\kappa$-terms $w_{\mathrm{g}, 3}$ verifying the conditions:

s3.1) all bases of $w_{\mathrm{g}, 3}$ are Lyndon words of length at most $k$;

s3.2) for each edge e $\in \Gamma$, the $\kappa$-terms $w_{\alpha(\mathrm{e}), 3} w_{\mathrm{e}, 3}$ and $w_{\omega(\mathrm{e}), 3}$ have the same bases;

s3.3) for each edge e $\in \Gamma, \mathbf{L S l}_{n} \models w_{\alpha(\mathrm{e}), 3} w_{\mathrm{e}, 3}=w_{\omega(\mathrm{e}), 3}$. That is, $\delta_{3}^{\prime}$ is a $\kappa$-solution over $\mathbf{L} \mathbf{S l}_{n}$;

s3.4) for all vertices $\mathrm{v}, \mathrm{w} \in \Gamma$ such that $\mathbf{L S l}_{N} \models w_{\mathrm{v}, 0}=w_{\mathrm{w}, 0}$, the $\kappa$-terms $w_{\mathrm{v}, 3}$ and $w_{\mathrm{w}, 3}$ have the same bases;

$\mathrm{s} 3.5)$ for all vertices $\mathrm{v}, \mathrm{w} \in \Gamma$ such that $\mathbf{L S l}_{N} \models w_{\mathrm{v}, 0}=w_{\mathrm{w}, 0}, \mathbf{L S l}_{n} \models w_{\mathrm{v}, 3}=w_{\mathrm{w}, 3}$;

s3.6) every factor of length $n$ of $\varepsilon\left(w_{\mathrm{g}, 2}\right)$ is also a factor of $\varepsilon\left(w_{\mathrm{g}, 3}\right)$;

s3.7) $w_{\mathrm{g}, 3}$ is of the form $w_{\mathrm{g}, 3}=l_{\mathrm{g}} w_{\mathrm{g}, 3}^{\prime} r_{\mathrm{g}}$.

In the process, the bases of $w_{\mathrm{g}, 2}$ are not lost and $w_{\mathrm{g}, 3}$ gets the bases $v$ that were missing. The following cases may arise.

Case 1. $v$ is a basis of $w_{\alpha(\mathrm{e}), 2} w_{\mathrm{e}, 2}$ and it is not a basis of $w_{\omega(\mathrm{e}), 2}$, for some edge e $\in \Gamma$. Then $v$ is a basis of $w_{\alpha(\mathrm{e}), 2}$ or $w_{\mathrm{e}, 2}$, and we need to create the basis $v$ in $w_{\omega(\mathrm{e}), 3}$. The basis $v$ was created by the transformations (6.2) in Step 1, where a certain occurrence of a subterm 
$u^{\omega+p}$ in $w_{\alpha(\mathrm{e}), 0}$ or $w_{\mathrm{e}, 0}$ was replaced by $\left(u_{1} \overline{u_{2}} u_{3}\right)^{\omega-p^{\prime}} u^{\ell_{u} p^{\prime}+p}$ with $\left|u_{2}\right|=k$. Notice that $\overline{u_{2}}$ is of the form $\overline{u_{2}}=a_{1} \cdots a_{i^{\prime}-1}\left(a_{i^{\prime}} \cdots a_{j} a_{i} \cdots a_{i^{\prime}-1}\right)^{\omega} a_{i^{\prime}} \cdots a_{k}$, whence $v=a_{i^{\prime}} \cdots a_{j} a_{i} \cdots a_{i^{\prime}-1}$. Suppose that $u^{\ell_{u}}=z_{q}$ for some $z_{q}$ defined in Step 1. Recall that $z_{q}^{\prime}=u_{q, 1} u_{q, 2} u_{q, 3}$ is a factor of $z_{q}$, with $\left|u_{q, 1}\right|=\left|u_{q, 3}\right|=n$ and $u_{q, 2}=u_{2}$, whence $\left|z_{q}^{\prime}\right|=2 n+k$. We may assume, without loss of generality, that $z_{q}^{\prime}$ can be extended to a factor $z_{q}^{\prime \prime}=u_{q, 0} z_{q}^{\prime} u_{q, 4}$ of $z_{q}$, with $\left|z_{q}^{\prime \prime}\right|=N$ and $\left|u_{q, 0}\right|,\left|u_{q, 4}\right| \geq 3 n$. Therefore $z_{q}^{\prime \prime}$ is a factor of $\varepsilon\left(w_{\alpha(\mathrm{e}), 0} w_{\mathrm{e}, 0}\right)$ of length $N$, whence $z_{q}^{\prime \prime}$ is also a factor of $\varepsilon\left(w_{\omega(\mathrm{e}), 0}\right)$ since $\delta_{0}^{\prime}$ is a $\kappa$-solution over $\mathbf{L S l}_{N}$ by Lemma 5.8. So, $z_{q}^{\prime \prime}$ is a factor of the $\kappa$-term $w_{\omega(\mathrm{e}), 2}$ by s1.2) and s2.5). Therefore, we may choose an occurrence of the factor $z_{q}^{\prime \prime}$ in $w_{\omega(\mathrm{e}), 2}$ and replace it by $u_{q, 0} u_{q, 1} \overline{u_{q, 2}} u_{q, 3} u_{q, 4}$, thus creating the basis $v$ in $w_{\omega(\mathrm{e}), 3}$. Notice that this makes also appear in $w_{\omega(\mathrm{e}), 3}$ all the eventual new factors of length $n$ that were created in $w_{\alpha(\mathrm{e}), 1} w_{\mathrm{e}, 1}$ when the subterm $u^{\omega+p}$ in $w_{\alpha(\mathrm{e}), 0} w_{\mathrm{e}, 0}$ was replaced by $\left(u_{1} \overline{u_{2}} u_{3}\right)^{\omega-p^{\prime}} u^{\ell_{u} p^{\prime}+p}$. The need to make appear these new factors is to obtain condition s3.3). Notice on the other hand that the transformation $u_{q, 1} u_{q, 2} u_{q, 3} \rightarrow u_{q, 1} \overline{u_{q, 2}} u_{q, 3}$ may, eventually, make some factor $u^{\prime}$ of length $n$ of $u_{q, 1} u_{q, 2} u_{q, 3}$ to be lost. Since $n \leq N$, condition s1.2) states that that transformation in $w_{\alpha(\mathrm{e}), 0} w_{\mathrm{e}, 0}$ does not make $u^{\prime}$ to be lost (because $u^{\prime}$ has other occurrences in $\left.w_{\alpha(\mathrm{e}), 0} w_{\mathrm{e}, 0}\right)$. However, that transformation in $w_{\omega(\mathrm{e}), 2}$ could make the factor $u^{\prime}$ disappear. To avoid this to happen notice that, by the remark after Definition 5.6, we may assume that $z_{q}^{\prime}=u_{q, 1} u_{q, 2} u_{q, 3}$ has at least two disjoint occurrences in $z_{q}^{\prime \prime}$. Since we only transform one of the occurrences of $z_{q}^{\prime}$, it follows that $u^{\prime}$ is also not lost in the $w_{\omega(\mathrm{e}), 2}$ case.

We should also remark that the above procedure can be applied for all possible bases $v$ in the above conditions, without conflicting one with the others (or with cases 2 and 3 below). Indeed, suppose that $v_{1}$ and $v_{2}$ are two distinct bases that were originated, respectively, by transformations $u_{m, 1} u_{m, 2} u_{m, 3} \rightarrow u_{m, 1} \overline{u_{m, 2}} u_{m, 3}$ and $u_{q, 1} u_{q, 2} u_{q, 3} \rightarrow u_{q, 1} \overline{u_{q, 2}} u_{q, 3}$ with $m<q$. The occurrences of $z_{m}^{\prime}=u_{m, 1} u_{m, 2} u_{m, 3}$ and $z_{q}^{\prime}=u_{q, 1} u_{q, 2} u_{q, 3}$ where those transformations should be performed in $w_{\omega(\mathrm{e}), 2}$ are necessarily disjoint by condition (6.3). Indeed, since $v_{1} \neq v_{2}, z_{q}^{\prime} \neq z_{m}^{\prime}$ and so $z_{m}^{\prime}$ is not a factor of $z_{q}$. Therefore $z_{m}^{\prime}$ is not a factor of the factor $z_{q}^{\prime \prime}=u_{q, 0} z_{q}^{\prime} u_{q, 4}$ of $z_{q}$. Since both $u_{q, 0}$ and $u_{q, 4}$ have length at least $3 n$, it follows that the occurrences of $z_{m}^{\prime}$ and $z_{q}^{\prime}$, where the transformations are to be performed in $w_{\omega(\mathrm{e}), 2}$, are disjoint. Moreover those occurrences of $z_{m}^{\prime}$ and $z_{q}^{\prime}$ are also disjoint from the second occurrences of $z_{q}^{\prime}$ and $z_{m}^{\prime}$ in $z_{q}^{\prime \prime}$ and $z_{m}^{\prime \prime}$, respectively, where the transformations are not applied.

Case 2. $v$ is a basis of $w_{\omega(\mathrm{e}), 2}$ and it is not a basis of $w_{\alpha(\mathrm{e}), 2} w_{\mathrm{e}, 2}$, for some edge e $\in \Gamma$. This case can be treated like the previous one. The only difference is that we find an occurrence of the extension $z_{q}^{\prime \prime}=u_{q, 0} z_{q}^{\prime} u_{q, 4}$ in: 1) exactly one of $w_{\alpha(\mathrm{e}), 2}$ and $\left.w_{\mathrm{e}, 2} ; 2\right)$ both of $w_{\alpha(\mathrm{e}), 2}$ and $\left.w_{\mathrm{e}, 2} ; 3\right)$ none of $w_{\alpha(\mathrm{e}), 2}$ and $w_{\mathrm{e}, 2}$. In the first case we replace the occurrence of $z_{q}^{\prime \prime}$ by $u_{q, 0} u_{q, 1} \overline{u_{q, 2}} u_{q, 3} u_{q, 4}$, thus creating the basis $v$ in $w_{\alpha(\mathrm{e}), 2} w_{\mathrm{e}, 2}$. In the second case we also replace $z_{q}^{\prime \prime}$ by $u_{q, 0} u_{q, 1} \overline{u_{q, 2}} u_{q, 3} u_{q, 4}$, but it suffices to make that only in $w_{\mathrm{e}, 2}$. In the third case, $z_{q}^{\prime \prime}$ has an occurrence in $w_{\alpha(\mathrm{e}), 2} w_{\mathrm{e}, 2}$ overlapping both $w_{\alpha(\mathrm{e}), 2}$ and $w_{\mathrm{e}, 2}$. Since $z_{q}^{\prime}$ has two disjoint occurrences in $z_{q}^{\prime \prime}$, by the remark after Definition 5.6, and at least one of them does not overlap the factor $c_{\mathrm{e}}$ of $w_{\alpha(\mathrm{e}), 2} w_{\mathrm{e}, 2}$, where $c_{\mathrm{e}}=r_{\alpha(\mathrm{e})} l_{\mathrm{e}}$ is the center of edge e, we may replace that occurrence of $z_{q}^{\prime}$ by $u_{q, 1} \overline{u_{q, 2}} u_{q, 3}$. 
Case 3. $v$ is a basis of $w_{\mathrm{v}, 2}$ and it is not a basis of $w_{\mathrm{w}, 2}$, where $\mathrm{v}, \mathrm{w} \in \mathcal{V}(\Gamma)$ are such that $\mathbf{L S l}_{N} \models w_{\mathrm{v}, 0}=w_{\mathrm{w}, 0}$. As in the previous cases, we could find an occurrence of some extension $z_{q}^{\prime \prime}$ of $z_{q}^{\prime}$ in $w_{\mathrm{w}, 2}$ and replace it by $u_{q, 0} u_{q, 1} \overline{u_{q, 2}} u_{q, 3} u_{q, 4}$. This would make also appear in $w_{\mathrm{w}, 3}$ all the eventual new factors of $w_{\mathrm{v}, 2}$ of length $n$, and would guarantee that condition s3.5) holds.

For each $\mathrm{g} \in \Gamma$, we denote by $w_{\mathrm{g}, 2}(1)$ the $\kappa$-term that arises from $w_{\mathrm{g}, 2}$ after all the above transformations. Since $w_{\mathrm{g}, 2}(1)$ may have some bases that were not present in $w_{\mathrm{g}, 2}$, the above procedure must be iterated until cases $1-3$ do not occur. Notice that the iteration must end indeed since the bases $v$ have length at most $k$ and there exists only a finite number of those bases. We denote by $w_{\mathrm{g}, 3}$ the $\kappa$-term thus obtained. That $w_{\mathrm{g}, 3}$ verifies conditions s3.1), s3.2), s3.4), s3.6) and s3.7) should be clear from the observations made through the construction above. To show s3.3), let e be an edge of $\Gamma$. We claim that the $\kappa$-words $\varepsilon\left(w_{\alpha(\mathrm{e}), 3} w_{\mathrm{e}, 3}\right)$ and $\varepsilon\left(w_{\omega(\mathrm{e}), 3}\right)$ have the same factors of length $n$. To prove the claim, we begin by noticing that, by s1.2), s2.5) and s3.6), each factor of length $n$ of $\varepsilon\left(w_{\mathrm{g}, 0}\right)$ is also a factor of $\varepsilon\left(w_{\mathrm{g}, 3}\right)$ for every $\mathrm{g} \in \Gamma$. Let $u^{\prime}$ be a factor of $\varepsilon\left(w_{\alpha(\mathrm{e}), 3} w_{\mathrm{e}, 3}\right)$ of length $n$. Suppose first that $u^{\prime}$ is a factor of $\varepsilon\left(w_{\alpha(\mathrm{e}), 0} w_{\mathrm{e}, 0}\right)$. In this case, since $\delta_{0}^{\prime}$ is a solution over $\mathbf{L S l}_{n}$ it follows that $u^{\prime}$ is a factor of $\varepsilon\left(w_{\omega(\mathrm{e}), 0}\right)$, whence $u^{\prime}$ is also a factor of $\varepsilon\left(w_{\omega(\mathrm{e}), 3}\right)$. Suppose now that $u^{\prime}$ is not a factor of $\varepsilon\left(w_{\alpha(\mathrm{e}), 0} w_{\mathrm{e}, 0}\right)$. Then $u^{\prime}$ is a factor of $\varepsilon\left(w_{\alpha(\mathrm{e}), 3} w_{\mathrm{e}, 3}\right)$ because it was created in Step 1 or the beginning of Step 3 as a consequence of the application of some transformation $u_{q, 1} u_{q, 2} u_{q, 3} \rightarrow u_{q, 1} \overline{u_{q, 2}} u_{q, 3}$. But, as we saw above, the same transformation must have been applied to $w_{\omega(\mathrm{e}), 0}$ or $w_{\omega(\mathrm{e}), 2}$, thus creating the factor $u^{\prime}$ in $\varepsilon\left(w_{\omega(\mathrm{e}), 3}\right)$. The proof that every factor of length $n$ of $\varepsilon\left(w_{\omega(\mathrm{e}), 3}\right)$ is also a factor of $\varepsilon\left(w_{\alpha(\mathrm{e}), 3} w_{\mathrm{e}, 3}\right)$ is analogous and, so, we deduce the claim. To conclude s3.3) it suffices to note that $\varepsilon\left(w_{\omega(\mathrm{e}), 3}\right)$ and $\varepsilon\left(w_{\alpha(\mathrm{e}), 3} w_{\mathrm{e}, 3}\right)$ have the same prefix and the same suffix of length $n-1$ since $\delta_{0}^{\prime}$ is a solution over $\mathbf{L S l}_{N}, n \leq N$ and Steps 1 to 3 do not change the prefix neither the suffix of length $n$ of any $\varepsilon\left(w_{\mathrm{g}, 0}\right)$. Finally, condition s3.5) can be shown analogously.

\subsection{Final steps}

This subsection will be devoted to presenting the two final steps of the algorithm of construction of the $\kappa$-solution $\delta^{\prime}$ over LSl.

Step 4. We claim that is possible to derive from $w_{\mathrm{g}, 3}$ a $\kappa$-term $w_{\mathrm{g}, 4}$ with the following properties:

s4.1) $w_{\mathrm{g}, 4}$ verifies conditions $\left.\left.\mathrm{s} 3.1\right)-\mathrm{s} 3.7\right)$, that is, conditions $\mathrm{s} 3.1$ )-s3.7) hold with 4 in the place of 3 ;

s4.2) if $\pi^{\alpha}$ is a subterm of $w_{\mathrm{g}, 4}$, with $\pi$ an infinite $\kappa$-term and $\alpha \in \omega+\mathbb{Z}$, then $\pi=u^{\beta} \rho u^{\omega}$ for some $u \in A^{+}, \rho \in T_{A}^{\kappa}$ and $\beta \in \omega+\mathbb{Z}$. Moreover, every occurrence of $\pi^{\alpha}=\left(u^{\beta} \rho u^{\omega}\right)^{\alpha}$ in $w_{\mathrm{g}, 4}$ takes place in a subterm of the form $u^{\omega}\left(u^{\beta} \rho u^{\omega}\right)^{\alpha} u^{\beta}$.

Consider the occurrences of subterms of $w_{\mathrm{g}, 3}$ of the form $\pi^{\alpha}$, with $\pi$ and $\alpha$ infinite, and let $m=f\left(w_{\mathrm{g}, 3}\right)$ be the number of such occurrences that do not verify condition s4.2). We proceed by induction on $m$. The case $m=0$ is trivial: it suffices to take $w_{\mathrm{g}, 4}=w_{\mathrm{g}, 3}$. So we assume that $m \geq 1$ and, as induction hypothesis, that the claim is valid for all $\kappa$-terms $w$ 
such that $f(w)<m$ and $w$ verifies conditions s3.1)-s3.7). Let $\pi_{1}^{\alpha_{1}}$, with $\pi_{1}$ and $\alpha_{1}$ infinite, be an occurrence in $w_{\mathrm{g}, 3}$ not verifying condition s4.2). As $\pi_{1}$ is infinite, it is of the form $\pi_{1}=y_{1} \pi_{2}^{\alpha_{2}} y_{2}$ for some $y_{1}, \pi_{2}, y_{2} \in T_{A}^{\kappa}$ and $\alpha_{2} \in \omega+\mathbb{Z}$, with $y_{1}$ and $y_{2}$ possibly empty but not simultaneously. We proceed by induction on the rank $r$ of $\pi_{1}^{\alpha_{1}}$. Assume first that $r=2$, so that $\pi_{2}=u \in A^{+}$and $\pi_{1}^{\alpha_{1}}=\left(y_{1} u^{\alpha_{2}} y_{2}\right)^{\alpha_{1}}$. We apply one right-left transformation of type 3 and one transformation of type 5 , to change the occurrence $\pi_{1}^{\alpha_{1}}$ in $w_{\mathrm{g}, 3}$ as follows

$$
\pi_{1}^{\alpha_{1}}=\left(y_{1} u^{\alpha_{2}} y_{2}\right)^{\alpha_{1}} \rightarrow\left(y_{1} u^{\omega} u^{\alpha_{2}} y_{2}\right)^{\alpha_{1}} \rightarrow y_{1} u^{\omega}\left(u^{\alpha_{2}} y_{2} y_{1} u^{\omega}\right)^{\alpha_{1}-1} u^{\alpha_{2}} y_{2},
$$

and denote by $w_{\mathrm{g}, 3}(1)$ the $\kappa$-term thus produced. Notice that $f\left(w_{\mathrm{g}, 3}(1)\right)=m-1$ and $w_{\mathrm{g}, 3}(1)$ verifies conditions s3.1)-s3.7). So, the claim follows from the induction hypothesis on $m$. Suppose now that $r>2$ and, as induction hypothesis, that condition s4.2) holds for all subterms of the form $\pi^{\alpha}$ of rank less than $r$. Hence, as $\pi_{2}^{\alpha_{2}}$ is a subterm of $\pi_{1}, \pi_{2}^{\alpha_{2}}$ is a $\kappa$-term of rank less than $r$. So, by the induction hypothesis on $r$, we deduce that $y_{1}=y_{1}^{\prime} u^{\omega}$, $\pi_{2}=u^{\beta} \rho u^{\omega}$ and $y_{2}=u^{\beta} y_{2}^{\prime}$ for some $u \in A^{+}, \rho, y_{1}^{\prime}, y_{2}^{\prime} \in T_{A}^{\kappa}$ and $\beta \in \omega+\mathbb{Z}$. In this case, as above, we may transform the occurrence $\pi_{1}^{\alpha_{1}}$ in $w_{\mathrm{g}, 3}$ as follows

$$
\pi_{1}^{\alpha_{1}}=\left(y_{1}^{\prime} u^{\omega} \pi_{2}^{\alpha_{2}} u^{\beta} y_{2}^{\prime}\right)^{\alpha_{1}} \rightarrow\left(y_{1}^{\prime} u^{\omega} u^{\omega} \pi_{2}^{\alpha_{2}} u^{\beta} y_{2}^{\prime}\right)^{\alpha_{1}} \rightarrow y_{1}^{\prime} u^{\omega}\left(u^{\omega} \pi_{2}^{\alpha_{2}} u^{\beta} y_{2}^{\prime} y_{1}^{\prime} u^{\omega}\right)^{\alpha_{1}-1} u^{\omega} \pi_{2}^{\alpha_{2}} u^{\beta} y_{2}^{\prime},
$$

and denote by $w_{\mathrm{g}, 3}(1)$ the $\kappa$-term thus produced. Also in this case, we have that $f\left(w_{\mathrm{g}, 3}(1)\right)=$ $m-1$ and $w_{\mathrm{g}, 3}(1)$ verifies conditions s3.1)-s3.7), whence the claim follows from the induction hypothesis on $m$. This completes the proof of the claim. Notice that the proof is constructive, whence the $\kappa$-term $w_{\mathrm{g}, 4}$ is effectively computable.

Step 5. This is the last step of the algorithm. Our first objective is to reduce the problem to consider only rank $1 \kappa$-terms. For each $\mathrm{g} \in \Gamma$, let $w_{\mathrm{g}, \diamond}$ be the rank $1 \kappa$-term obtained from $w_{\mathrm{g}, 4}$ by the elimination of all the infinite exponents of powers in which the base is an infinite $\kappa$-term. That is, $w_{\mathrm{g}, \diamond}$ is the $\kappa$-term produced when we apply all possible transformations $x^{\omega+i} \rightarrow x$ with $x$ an infinite $\kappa$-term. Notice that this transformation is not derivable from the rewriting rules, whence $w_{\mathrm{g}, \diamond}$ is not a derivation from $w_{\mathrm{g}, 4}$. Therefore, the pseudovariety $\mathbf{V}$ and the finite semigroup $S$ may not verify $w_{\mathrm{g}, 4}=w_{\mathrm{g}, \diamond}$. However in the following procedure we will revert the above transformation (that is, we will put each exponent in the exact position where it was before) thus recovering the value of $w_{\mathrm{g}, 4}$ over $\mathbf{V}$ and $S$. Since $l_{\mathrm{g}}$ and $r_{\mathrm{g}}$ are respectively a prefix and a suffix of $w_{\mathrm{g}, 4}$, by s4.1) and $\left.\mathrm{s} 3.7\right), w_{\mathrm{g}, \diamond}$ can be written as

$$
w_{\mathrm{g}, \diamond}=l_{\mathrm{g}} u_{\mathrm{g}, 0}^{\prime} u_{\mathrm{g}, 1}^{\alpha_{\mathrm{g}, 1}} u_{\mathrm{g}, 1}^{\prime} u_{\mathrm{g}, 2}^{\alpha_{\mathrm{g}, 2}} \cdots u_{\mathrm{g}, m_{\mathrm{g}}-1}^{\prime} u_{\mathrm{g}, m_{\mathrm{g}}}^{\alpha_{\mathrm{g}}} u_{\mathrm{g}, m_{\mathrm{g}}}^{\prime} r_{\mathrm{g}}
$$

with $m_{\mathrm{g}} \geq 1, u_{\mathrm{g}, 0}^{\prime}, \ldots, u_{\mathrm{g}, m_{\mathrm{g}}}^{\prime} \in A^{*}, u_{\mathrm{g}, 1}, \ldots, u_{\mathrm{g}, m_{\mathrm{g}}} \in A^{+}$and $\alpha_{\mathrm{g}, 1}, \ldots, \alpha_{\mathrm{g}, m_{\mathrm{g}}} \in \omega+\mathbb{Z}$. Let $\delta_{\diamond}: \Gamma \rightarrow \Omega_{A}^{\kappa} \mathbf{S}$ be the labeling of $\Gamma$ defined by $\delta_{\diamond}(\mathrm{g})=\varepsilon\left(w_{\mathrm{g}, \diamond}\right)$ for any $\mathrm{g} \in \Gamma$ and let $\gamma_{\diamond}=\psi \circ \delta_{\diamond}$. In view of the form of the $\kappa$-terms $w_{\mathrm{g}, 4}$ given by condition s4.2), it is clear that $\mathbf{L S l}_{n} \models w_{\mathrm{g}, \diamond}=w_{\mathrm{g}, 4}$. Since $\delta_{4}^{\prime}$ is a solution of $\Gamma$ over $\mathbf{L} \mathbf{S l}_{n}$ with respect to $\left(\gamma_{0}, \psi\right)$, we deduce that $\delta_{\diamond}$ is a solution of $\Gamma$ over $\mathbf{L} \mathbf{S l}_{n}$ with respect to $\left(\gamma_{\diamond}, \psi\right)$.

Claim 6.2 It is possible to derive from $w_{\mathrm{g}, \diamond}$ a (rank 1) $\kappa$-term $\widehat{w_{\mathrm{g}, \diamond}}$ of the form

$$
\widehat{w_{\mathrm{g}, \diamond}}=\widehat{l_{\mathrm{g}}} u_{\mathrm{g}, 0}^{\prime \prime} u_{\mathrm{g}, 1}^{\alpha_{\mathrm{g}, 1}} u_{\mathrm{g}, 1}^{\prime \prime} u_{\mathrm{g}, 2}^{\alpha_{\mathrm{g}, 2}} \cdots u_{\mathrm{g}, m_{\mathrm{g}}-1}^{\prime \prime} u_{\mathrm{g}, m_{\mathrm{g}}}^{\alpha_{\mathrm{g}}} u_{\mathrm{g}, m_{\mathrm{g}}}^{\prime \prime} \widehat{r_{\mathrm{g}}}
$$

such that the labeling $\delta_{\diamond}^{\prime}: \Gamma \rightarrow \Omega_{A}^{\kappa} \mathbf{S}$ defined by $\delta_{\diamond}^{\prime}(\mathrm{g})=\varepsilon\left(\widehat{w_{\mathrm{g}, \diamond}}\right)$, for any $\mathrm{g} \in \Gamma$, is a $\kappa$-solution of $\Gamma$ over LSl with respect to $\left(\gamma_{\diamond}, \psi\right)$ that verifies condition (ST.3). 
This claim will be proved below. For now assume the claim is true and notice that, apart the substitutions of $l_{\mathrm{g}}$ and $r_{\mathrm{g}}$ by $\widehat{l_{\mathrm{g}}}$ and $\widehat{r_{\mathrm{g}}}$, respectively, all the transformations occur in the factors $u_{\mathrm{g}, j}^{\prime}$. So, by condition s4.2), to restore the exponents that were eliminated in the formation of $w_{\mathrm{g}, \diamond}$, it suffices to search in $\widehat{w_{\mathrm{g}, \diamond}}$ for the infinite powers $u_{\mathrm{g}, j}^{\alpha_{\mathrm{g}, j}}$ that mark the positions where the exponents were in $w_{\mathrm{g}, 4}$ and add them in $\widehat{w_{\mathrm{g}, \diamond}}$ at the same positions. Denote by $w_{\mathrm{g}, 5}$ the $\kappa$-term thus produced. This finishes the construction of $\delta^{\prime}$ which, as one recalls, is defined as follows.

Definition 6.3 (labeling $\delta^{\prime}$ ) The labeling $\delta^{\prime}$ of $\Gamma$ by $\kappa$-words of $\Omega_{A}^{\kappa} \mathbf{S}$ is defined by $\delta^{\prime}(\mathrm{g})=$ $\varepsilon\left(w_{\mathrm{g}, 5}\right)$ for any $\mathrm{g} \in \Gamma$.

Due to the special form of the $\kappa$-terms $w_{\mathrm{g}, 4}$ yielded by s4.2), it is clear that $\widehat{w_{\mathrm{g}, \diamond}}$ and $w_{\mathrm{g}, 5}$ are equal over LSl since they have the same prefix $\widehat{l_{\mathrm{g}}}$, the same suffix $\widehat{r_{\mathrm{g}}}$ and the same non-periodic bi-infinite factors. Moreover, $w_{\mathrm{g}, 5}$ has recovered the value of $w_{\mathrm{g}, 4}$ over $\mathbf{V}$ and $S$. Hence, $\delta^{\prime}$ is a $\kappa$-solution of $\Gamma$ over $\mathbf{L S l}$ with respect to $\left(\gamma_{0}, \psi\right)$ that verifies conditions (ST.3) and (ST.4). Furthermore, as referred in Remark 6.1, $\delta^{\prime}$ satisfies also conditions (ST.1) and (ST.2). Therefore, to establish the ST $\kappa$-reducibility of $\mathbf{L S l} \vee \mathbf{V}$ and deduce the $\kappa$-reducibility of $\mathbf{L S l} \vee \mathbf{V}$, it remains to prove the above claim.

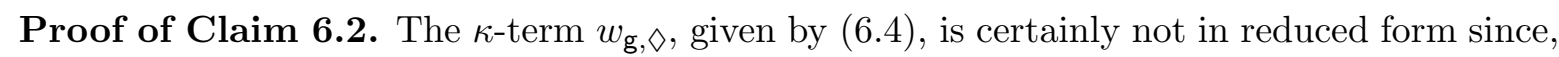
by $\mathrm{s} 4.2)$, it contains factors of the form $u^{\omega} u^{\beta}$. However, to reduce it, it suffices to apply leftright transformations of types 4 and 3 to replace all possible subterms of the form $u^{\omega+i} u^{\ell} u^{\omega+j}$ by $u^{\omega+i+\ell+j}$. So, we will assume that the factorization s4.2) is in reduced form. Since the powers $u^{\omega+i+\ell+j}$ will be preserved, we can then replace them by $u^{\omega+i} u^{\ell} u^{\omega+j}$ by using the dual right-left transformations of types 4 and 3. Notice that we can also assume, without loss of generality, that, for each $j \in\left\{1, \ldots, m_{\mathrm{g}}\right\}, u_{\mathrm{g}, j}^{2 n}$ is a suffix of $u_{\mathrm{g}, j-1}^{\prime}$ and a prefix of $u_{\mathrm{g}, j}^{\prime}$.

We employ the technique introduced in [17, Subsection 6.5] and also used in [16, Subsection 6.5]. The motivations and justifications of the procedure will be omitted since they are given in full detail in those papers. Since that procedure applies only to finite words, we begin by associating to $w_{\mathrm{g}, \diamond}$ the finite word $w_{\mathrm{g}, \nabla}$ obtained from $w_{\mathrm{g}, \diamond}$ by the elimination of all exponents $\alpha_{\mathrm{g}, j}$,

$$
w_{\mathrm{g}, \nabla}=l_{\mathrm{g}} u_{\mathrm{g}, 0}^{\prime} u_{\mathrm{g}, 1} u_{\mathrm{g}, 1}^{\prime} u_{\mathrm{g}, 2} \cdots u_{\mathrm{g}, m_{\mathrm{g}}-1}^{\prime} u_{\mathrm{g}, m_{\mathrm{g}}} u_{\mathrm{g}, m_{\mathrm{g}}}^{\prime} r_{\mathrm{g}} .
$$

Notice that this transformation is not derivable from the rewriting rules. In particular the pseudovariety $\mathbf{V}$ and the finite semigroup $S$ may not verify $w_{\mathrm{g}, \diamond}=w_{\mathrm{g}, \nabla}$. However, once again, we will revert the above transformation (that is, we will put each exponent $\alpha_{\mathrm{g}, j}$ in the exact position where it was before) thus recovering the value of $w_{\mathrm{g}, \diamond}$ over $\mathbf{V}$ and $S$. Let $\delta_{\nabla}: \Gamma \rightarrow \Omega_{A}^{\kappa} \mathbf{S}$ be the labeling of $\Gamma$ defined by $\delta_{\nabla}(\mathrm{g})=w_{\mathrm{g}, \nabla}$ for any $\mathrm{g} \in \Gamma$ and let $\gamma_{\nabla}=\psi \circ \delta_{\nabla}$. It is clear that $\mathbf{L S l}_{n} \models w_{\mathrm{g}, \nabla}=w_{\mathrm{g}, \diamond}$. Since $\delta_{\diamond}$ is a solution of $\Gamma$ over $\mathbf{L S l}_{n}$ with respect to $\left(\gamma_{\diamond}, \psi\right)$, we deduce that $\delta_{\nabla}$ is a solution of $\Gamma$ over $\mathbf{L S l}_{n}$ with respect to $\left(\gamma_{\nabla}, \psi\right)$. We are now ready to apply the procedure of $[17$, Subsection 6.5$]$ to each word $w_{\mathrm{g}, \nabla}$. We notice that the only rule that that procedure uses is (R.6).

As shown in [17], there exists a factorization

$$
w_{\mathrm{g}, \nabla}=l_{\mathrm{g}} z_{\mathrm{g}, 0} c_{\mathrm{g}, 1} z_{\mathrm{g}, 1} c_{\mathrm{g}, 2} \cdots c_{\mathrm{g}, j_{\mathrm{g}}} z_{\mathrm{g}, j_{\mathrm{g}}} r_{\mathrm{g}}
$$

of $w_{\mathrm{g}, \nabla}$ such that $j_{\mathrm{g}} \geq 0$ and 
- $c_{\mathrm{g}, 1}, \ldots, c_{\mathrm{g}, j_{\mathrm{g}}} \in C_{F}$ are centers of $F_{\Gamma}$;

- $z_{\mathrm{g}, 0}, z_{\mathrm{g}, 1}, \ldots, z_{\mathrm{g}, j_{\mathrm{g}}} \in A^{+}$with $\left|z_{\mathrm{g}, i}\right| \geq 3 k-1$;

- the factorization contains all allowed occurrences of non-periodic centers of $F_{\Gamma}$ in $w_{\mathrm{g}, \nabla}$;

- if $c_{\mathrm{f}}$ is a periodic center of $F_{\Gamma}$ that occurs in $w_{\mathrm{g}, \nabla}$, then the factorization contains exactly one occurrence of $c_{\mathrm{f}}$.

We then use factorization (6.7) to transform $w_{\mathrm{g}, \nabla}$ into the following $\kappa$-term

$$
w_{\mathrm{g}, \nabla}(1)=\widehat{l_{\mathrm{g}}} z_{\mathrm{g}, 0} \widehat{\mathrm{g}_{\mathrm{g}, 1}} z_{\mathrm{g}, 1} \widehat{c_{\mathrm{g}, 2}} \cdots \widehat{c_{\mathrm{g}, j_{\mathrm{g}}}} z_{\mathrm{g}, j_{\mathrm{g}}} \widehat{r_{\mathrm{g}}}
$$

where each $\widehat{c_{\mathrm{g}, \ell}}$ is a rank $1 \kappa$-term defined in (5.8).

We proceed with the transformation of each word $z_{\mathrm{g}, \ell}\left(\ell \in\left\{0, \ldots, j_{\mathrm{g}}\right\}\right)$ in factorization (6.8), into a rank $1 \kappa$-term $\widehat{z_{\mathrm{g}, \ell}}$. This will be done in four substeps.

Step 5.1. Consider the marked factorization, described in Subsection 4.1,

$$
z_{\mathrm{g}, \ell}=z_{0} v_{1} z_{1} v_{2} \cdots v_{q} z_{q}
$$

of $z_{\mathrm{g}, \ell}$. By definition of marked factors, if $q \geq 1$, then $\left|v_{i}\right| \geq 2 k$ for every $i \in\{1, \ldots, q\}$, so that we can write $v_{i}=v_{i, 1} v_{i, 2} v_{i, 3}$ for some words $v_{i, 1}, v_{i, 2}$ and $v_{i, 3}$ with $\left|v_{i, 1}\right|=\left|v_{i, 3}\right|=k$. We define $\widehat{v_{i}}=\overline{v_{i, 1}} v_{i, 2} \overline{v_{i, 3}}$, and let $z_{\mathrm{g}, \ell}(1)$ be the following rank $1 \kappa$-term

$$
z_{\mathrm{g}, \ell}(1)=z_{0} \widehat{v_{1}} z_{1} \widehat{v_{2}} z_{2} \cdots \widehat{v_{q}} z_{q} .
$$

Step 5.2. By definition of free factors, if $z_{0}$ is not the empty word, then it has length greater than $k$. In this case, we define $\widehat{z_{0}}=\overline{z_{0,1}} z_{0,2}$ where $z_{0,1}$ is the prefix of length $k$ of $z_{0}$ and $z_{0}=z_{0,1} z_{0,2}$. Symmetrically, if $z_{q}$ is not the empty word, we let $\widehat{z_{q}}=z_{q, 1} \overline{z_{q, 2}}$ where $z_{q, 2}$ is the suffix of length $k$ of $z_{q}$ and $z_{q}=z_{q, 1} z_{q, 2}$. Now, let

$$
z_{\mathrm{g}, \ell}(2)=\widehat{z_{0}} \widehat{v_{1}} z_{1} \widehat{v_{2}} z_{2} \cdots \widehat{v_{q}} \widehat{z_{q}} \text {. }
$$

Step 5.3. Let $y \in A^{+}$be a factor of $z_{\mathrm{g}, \ell}$ such that $2 k \leq|y|<3 k$. Two cases may arise.

(Case I) Every extension of length $3 k-1$ in $z_{\mathrm{g}, \ell}$, of an occurrence of $y$, is a marked occurrence (see Subsection 4.1). In this case every occurrence of $y$ in $z_{\mathrm{g}, \ell}$ is contained in the marked factors $v_{i}$.

(Case II) There exists a free occurrence in $z_{\mathrm{g}, \ell}$ of an extension $\tilde{y}$, of length $3 k-1$, of an occurrence of $y$. In this case, by definition of free occurrence (cf. Subsection 4.1), there exists a $k^{\prime \prime}$-neighborhood $v$ of $\tilde{y}$ such that $v$ is $k^{\prime}$-abundant. In particular, $\operatorname{docc}(\tilde{y}, v) \geq k^{\prime}$. Furthermore, every occurrence of a factor of length $3 k-1$ in the $k^{\prime \prime}$-neighborhood $v$ is free. This means that at least $k^{\prime}-2$ of the disjoint occurrences of $\tilde{y}$ in $v$ occur disjoint from the marked factors. More precisely, there exists an integer $0 \leq i \leq q$ such that $\operatorname{docc}\left(\tilde{y}, z_{i}\right) \geq k^{\prime}-2$. Hence, since $y$ is a factor of $\tilde{y}$, $\operatorname{docc}\left(y, z_{i}\right) \geq k^{\prime}-2$. In this case, we say that $y$ has a free occurrence in $z_{\mathrm{g}, \ell}$. 
Consider the set $F$ of all factors $y$ of $z_{\mathrm{g}, \ell}$ such that $2 k \leq|y|<3 k$ and $y$ has a free occurrence in $z_{\mathrm{g}, \ell}$. By the second case above, for each $y \in F$ there exists an integer $0 \leq i \leq q$ such that $\operatorname{docc}\left(y, z_{i}\right) \geq k^{\prime}-2$. Therefore, the choice of $k^{\prime}$ allows us to select an occurrence for each $y \in F$ in such a way that these occurrences are pairwise disjoint. These occurrences are selected in the factors $z_{i}$ and, if $i=0$ or $i=q$, then we can select them, respectively, in $z_{0,2}$ and in $z_{q, 1}$. Since $2 k \leq|y|<3 k$, we can write $y=y_{1} y_{2} y_{3}$ for some words $y_{1}, y_{2}$ and $y_{3}$ with $\left|y_{1}\right|=\left|y_{3}\right|=k$. We substitute in $z_{\mathrm{g}, \ell}(2)$ the selected occurrence of $y$ by $\widehat{y}=\overline{y_{1}} y_{2} \overline{y_{3}}$. We then obtain a term $z_{\mathrm{g}, \ell}(3)$.

Adjusting Step Up to here, we have followed step by step the algorithm of transformation of $w_{\mathrm{g}, \nabla}$ given in [17]. Now, as announced above, we have to make a small adjustment on the procedure in order to revert the transformation that erased the exponents $\alpha_{\mathbf{g}, j}$ in $w_{\mathrm{g}, \diamond}$. Recall that by assumption $u_{\mathrm{g}, j}^{2 n}$ is a suffix of $u_{\mathrm{g}, j-1}^{\prime}$ and a prefix of $u_{\mathrm{g}, j}^{\prime}$, for every $j \in\left\{1, \ldots, m_{\mathrm{g}}\right\}$, whence the occurrence $u_{\mathrm{g}, j}$ in factorization (6.6) may be extended to an occurrence $u_{\mathrm{g}, j}^{4 n+1}$ in which the occurrence $u_{\mathrm{g}, j}$ of (6.6) is the central factor. On the other hand, no non-periodic center $c_{\mathrm{g}, \ell}$ occurs in $u_{\mathrm{g}, j}^{4 n+1}$. Indeed since $c_{\mathrm{g}, \ell}$ is as wide as we want, if $c_{\mathrm{g}, \ell}$ would be a factor of $u_{\mathrm{g}, j}^{4 n+1}$, then the bi-infinite words $\mathbf{w}_{\mathrm{g}}$ and $u_{\mathrm{g}, j}^{\infty}$ would be in the same orbit, which is impossible since the second one is periodic and the first one is not. Moreover, if $c_{\mathrm{g}, \ell}$ is a periodic center and it occurs in $u_{\mathrm{g}, j}^{4 n+1}$, we may assume without loss of generality that it occurs in the suffix $u_{\mathrm{g}, j}^{2 n}$. As a consequence, (we can assume that) $u_{\mathrm{g}, j}^{2 n+1}$ occurs in some factor $z_{\mathrm{g}, \ell}$ in factorization (6.8). Moreover, (we can assume that) $u_{\mathrm{g}, j}^{2 n+1}$ occurs in some free factor $z_{i}$ in factorization (6.9) of $z_{\mathrm{g}, \ell}$.

So, before applying the transformations foreseen for Step 5.3, we localize in $z_{i}$ the fixed occurrence of $u_{\mathrm{g}, j}$ that comes from (6.6). Then, we replace that occurrence of $u_{\mathrm{g}, j}$ by $u_{\mathrm{g}, j}^{\alpha_{\mathrm{g}, j}}$. Now, since we want to control the bi-infinite factors that are being produced, we consider the suffix $s_{\mathrm{g}, j-1}$ of $u_{\mathrm{g}, j-1}^{\prime}$ of length $k$ and the prefix $p_{\mathrm{g}, j}$ of $u_{\mathrm{g}, j}^{\prime}$ of length $k$. Then $s_{\mathrm{g}, j-1}$ and $p_{\mathrm{g}, j}$ occur adjacent to the fixed occurrence of $u_{\mathrm{g}, j}$, and we replace those occurrences respectively by $\overline{s_{\mathrm{g}, j-1}}$ and $\overline{p_{\mathrm{g}, j}}$. After these three substitutions, Step 5.3 can be carried out as described above, and we notice that the substitutions have no impact on Step 5.3 since they were made in a free factor and, so, all the elements from $F$ continue to have enough occurrences in $z_{\mathrm{g}, \ell}$. This means that all the bi-infinite factors that would be obtained without those three substitutions are still obtained with them. The unique difference is that it was introduced the factor $\overline{s_{\mathrm{g}, j-1}} u_{\mathrm{g}, j}^{\alpha_{\mathrm{g}, j}} \overline{p_{\mathrm{g}, j}}$ which may have created (at most two) new bi-infinite factors.

Step 5.4. In this step, we admit the substitution of any occurrence, in the factors of $z_{\mathrm{g}, \ell}(3)$ which were obtained from the transformations on the words $z_{i}$, of a factor $y \in A^{+}$of length $k$ by $\bar{y}$. We say that a term obtained from $z_{\mathrm{g}, \ell}(3)$ using these substitutions is irreducible when it is not possible to make more substitutions (that is, when there are no more occurrences of factors $y \in A^{+}$of length $k$ on the factors which resulted from substitutions on the words $z_{i}$ ). We choose an irreducible term and denote it by $\widehat{z_{\mathrm{g}, \ell}}$. This concludes the process of transformation of the word $z_{\mathrm{g}, \ell}$.

With this, we are able to complete the construction of the $\kappa$-term $\widehat{w_{\mathrm{g}, \diamond}}$, whose existence was 
stated in Claim 6.2. For that it suffices to consider the factorization (6.8) of $w_{\mathbf{g}, \nabla}(1)$ and let

$$
\widehat{w_{\mathrm{g}, \diamond}}=\widehat{l_{\mathrm{g}}} \widehat{z_{\mathrm{g}, 0}} \widehat{c_{\mathrm{g}, 1}} \widehat{z_{\mathrm{g}, 1}} \widehat{c_{\mathrm{g}, 2}} \cdots \widehat{c_{\mathrm{g}, j_{\mathrm{g}}}} \widehat{z_{\mathrm{g}, j_{\mathrm{g}}}} \widehat{r_{\mathrm{g}}}
$$

which is a rank $1 \kappa$-term that, by the Adjusting Step above, can be written in the form (6.5). Notice that the substitution of $u_{\mathrm{g}, j}$ by $u_{\mathrm{g}, j}^{\alpha_{\mathrm{g}, j}}$ in the Adjusting Step has recovered the value of $w_{\mathrm{g}, \diamond}$ over $\mathbf{V}$ and $S$.

To conclude the proof of Claim 6.2, we notice that $\delta_{\diamond}^{\prime}$ is a $\kappa$-solution of $\Gamma$ over LSl that satisfies condition (ST.3). We do not present here a direct proof of this statement since it would be a (lengthy) repetition of the arguments of the analogous proof presented in [17, Subsection 6.6] for the case of the pseudovariety LSl. Indeed, the algorithm of construction of the $\kappa$-solution $\delta^{\prime}$ over LSl, in [17], consists in associating to each $\mathrm{g} \in \Gamma$ a finite word $u_{\mathrm{g} \delta}$ such that $u_{\mathrm{g} \delta} \equiv_{n} \delta(\mathrm{g})$ and then apply to $u_{\mathrm{g} \delta}$ the procedure described above (to transform the word $w_{\mathrm{g}, \nabla}$, without the Adjusting Step) in such a way that $\delta^{\prime}$ verifies conditions (c1)(c4) of [17, Proposition 6.1]. These conditions (c1)-(c4) are essentially the ones that we have imposed here to our $\delta^{\prime}$, in the definition of the $\mathbf{L S l} \vee \mathbf{V}$ second type $\kappa$-reducibility. There are only two differences. In the first place, for the $\mathbf{L S I} \vee \mathbf{V}$ case, we have needed an extra condition, (ST.4), in order to deal with the pseudovariety V. Secondly, conditions (ST.1)(ST.4) refer to a solution $\delta_{0}^{\prime}$ over $\mathbf{L S l}_{N}$, while (c2)-(c4) refer to a solution $\delta$ over LSl. This difference is, however, merely apparent since in this paper we had to apply the $\kappa$-reducibility of $\mathbf{V}$ (thus losing the property of being a solution over LSl, but maintaining the property of being a solution over $\mathbf{L S l}_{N}$ ) before making the second type reduction (that is, the reduction to the case in which the labels of all the elements of $\Gamma$ are infinite), while in [17] it was made the reduction first (Proposition 6.1) and only then it was considered an "LSI ${ }_{n}$ approximation" given by the words $u_{\mathrm{g} \delta}$. We also notice that the need, in this paper, to begin the construction of $\delta^{\prime}$ with a solution $\delta_{0}^{\prime}$ over $\mathbf{L S l}_{N}$, for an integer $N$ larger than $n$, was because the starting $\kappa$-terms $w_{\mathrm{g}, 0}$ had arbitrary ranks and the transformations they suffered throughout steps 1 to 3 made the solution "decrease" to $\mathbf{L S l}_{n}$. So, basically, what we did in this paper was to seek for words $w_{\mathrm{g}, \nabla}$ that, as far as the pseudovariety LSI is concerned, were in the same conditions of the words $u_{\mathrm{g} \delta}$ in [17], so that we could apply to them the same algorithm (with the necessary adaptations, namely the Adjusting Step) of construction of a solution over LSl that, in particular, verifies condition (ST.3).

Observe that the introduction of the Adjusting Step made possible to have $\psi \circ \delta_{\diamond}^{\prime}=\gamma_{\diamond}$, a condition necessary to $\delta_{\diamond}^{\prime}$ being a solution with respect to $\left(\gamma_{\diamond}, \psi\right)$. On the other hand, as referred in the Adjusting Step, the only difference is that it created the factor $\overline{s_{\mathrm{g}, j-1}} u_{\mathrm{g}, j}^{\alpha_{\mathrm{g}, j}} \overline{p_{\mathrm{g}, j}}$ in $\widehat{w_{\mathrm{g}, \diamond}}$. But this is not a problem since by s4.1), s3.2) and s3.4): for each e $\in \mathcal{E}(\Gamma)$, the $\kappa$-terms $w_{\alpha(\mathrm{e}), 4} w_{\mathrm{e}, 4}$ and $w_{\omega(\mathrm{e}), 4}$ have the same bases; for every vertices $\mathrm{v}, \mathrm{w} \in \mathcal{V}(\Gamma)$ such that $\mathbf{L S l}_{N} \models w_{\mathrm{v}, 0}=w_{\mathrm{w}, 0}$, the $\kappa$-terms $w_{\mathrm{v}, 4}$ and $w_{\mathrm{w}, 4}$ have the same bases. Therefore, for instance, if $\mathrm{v}, \mathrm{w} \in \mathcal{V}(\Gamma)$ are such that $\mathbf{L S l}_{N}=w_{\mathrm{v}, 0}=w_{\mathrm{w}, 0}$ and a factor $\overline{s_{\mathrm{v}, j-1}} u_{\mathrm{v}, j}^{\alpha_{\mathrm{v}, j}} \overline{p_{\mathrm{v}, j}}$ was created in $\widehat{w_{\mathrm{v}, \diamond}}$, then $u_{\mathrm{v}, j}=u_{\mathrm{w}, j^{\prime}}, s_{\mathrm{v}, j-1}=s_{\mathrm{w}, j^{\prime}-1}$ and $p_{\mathrm{v}, j}=p_{\mathrm{w}, j^{\prime}}$ for some $j^{\prime}$. So, a factor $\overline{s_{\mathrm{w}, j^{\prime}-1}} u_{\mathrm{w}, j^{\prime}}^{\alpha_{\mathrm{w}, j^{\prime}}} \overline{p_{\mathrm{w}, j^{\prime}}}=\overline{s_{\mathrm{v}, j-1}} u_{\mathrm{v}, j} \alpha_{\mathrm{w}, j^{\prime}} \overline{p_{\mathrm{v}, j}}$ was created in $\widehat{w_{\mathrm{w}, \diamond}}$ thus introducing in $\widehat{w_{\mathrm{w}, \diamond}}$ the same (possibly) new bi-infinite factors that $\overline{s_{\mathrm{v}, j-1}} u_{\mathrm{v}, j}^{\alpha_{\mathrm{v}, j}} \overline{p_{\mathrm{v}, j}}$ has introduced in $\widehat{w_{\mathrm{v}, \diamond}}$. Therefore $\widehat{w_{\mathrm{v}, \diamond}}$ and $\widehat{w_{\mathrm{w}, \diamond}}$ have the same non-periodic bi-infinite factors and, as a consequence, $\delta_{\diamond}^{\prime}$ verifies condition (ST.3). Similarly, one could show that $\delta_{\diamond}^{\prime}$ is a solution over LSl and conclude that Claim 6.2 holds. 
This establishes the ST $\kappa$-reducibility of $\mathbf{L S I} \vee \mathbf{V}$, thus completing the proof of Theorem 5.1.

\section{Acknowledgements}

This work was supported, in part, by the European Regional Development Fund, through the programme COMPETE, and by the Portuguese Government through FCT - Fundação para a Ciência e a Tecnologia, under the project PEst-C/MAT/UI0013/2011. Both authors were also supported by FCT through the project PTDC/MAT/65481/2006, which was partly funded by the European Community Fund FEDER. The work leading to this paper has also been carried out within the framework of the ESF programme "Automata: from Mathematics to Applications (AutoMathA)", whose support is gratefully acknowledged.

\section{References}

[1] D. Albert, R. Baldinger and J. Rhodes, The identity problem for finite semigroups (the undecidability of), J. Symbolic Logic 57 (1992), 179-192.

[2] J. Almeida, Finite semigroups and universal algebra, (World Scientific, Singapore, 1995). English translation.

[3] J. Almeida, Finite semigroups: An Introduction to a unified theory of pseudovarieties, in Semigroups, Algorithms, Automata and Languages, ed. G. Gomes (World Scientific, 2002), pp. 3-64.

[4] J. Almeida and B. Steinberg, On the decidability of iterated semidirect products and applications to complexity, Proc. London Math. Soc. 80 (2000), 50-74.

[5] J. Almeida, J. C. Costa and M. Zeitoun, Tameness of pseudovariety joins involving R, Monatshefte für Mathematik 146 (2005), 89-111.

[6] J. Almeida, J. C. Costa and M. Zeitoun, Complete reducibility of systems of equations with respect to R, Portugalice Mathematica 64 (2007), 445-508.

[7] J. Almeida and M. Delgado, Tameness of the pseudovariety of abelian groups, Int. J. Algebra and Computation 15 (2005), 327-338.

[8] J. Almeida and P. G. Trotter, Hyperdecidability of pseudovarieties of orthogroups, Bull. Glasgow Math. J. 43 (2001), 67-83.

[9] J. Almeida and P. G. Trotter, The pseudoidentity problem and reducibility for completely regular semigroups, Bull. Austral. Math. Soc. 63 (2001), 407-433.

[10] J. Almeida and M. Zeitoun, Tameness of some locally trivial pseudovarieties, Communications in Algebra 31 (2003), 61-77.

[11] C. Ash, Inevitable graphs: a proof of the type II conjecture and some related decision procedures, Int. J. Algebra and Computation 1 (1991), 127-146. 
[12] K. Auinger and B. Steinberg, On the extension problem for partial permutations, Proc. Amer. Math. Soc. 131 (2003), 2693-2703.

[13] J. Brzozowski and I. Simon, Characterization of locally testable events, Discrete Math. 4 (1973), 243-271.

[14] J. C. Costa, Free profinite locally idempotent and locally commutative semigroups, Journal of Pure and Applied Algebra 163 (2001), 19-47.

[15] J. C. Costa, Reducibility of joins involving some locally trivial pseudovarieties, Communications in Algebra, 32 (2004), 3517-3535.

[16] J. C. Costa and C. Nogueira, Complete reducibility of the pseudovariety LSl, Int. J. Algebra and Computation 19 (2009), 247-282.

[17] J. C. Costa and M. L. Teixeira, Tameness of the pseudovariety LSl, Int. J. Algebra and Computation 14 (2004), 627-654.

[18] T. Coulbois and A. Khélif, Equations in free groups are not finitely approximable, Proc. Amer. Math. Soc. 127 (1999), 963-965.

[19] M. Lothaire, Algebraic combinatorics on words (Cambridge University Press, 2002).

[20] R. McNaughton, Algebraic decision procedures for local testability, Math. Systems Theory 8 (1974), 60-76.

[21] J. Reiterman, The Birkhoff theorem for finite algebras, Algebra Universalis 14 (1982), $1-10$.

[22] J. Rhodes, Undecidability, automata and pseudovarieties of semigroups, Int. J. Algebra and Computation 9 (1999), 455-473.

[23] J. Rhodes and B. Steinberg, The q-Theory of Finite Semigroups, (Springer Monographs in Mathematics, Springer, 2008).

[24] B. Steinberg, On algorithmic problems for joins of pseudovarieties, Semigroup Forum 62 (2001), 1-40.

[25] Y. Zalcstein, Locally testable semigroups, Semigroup Forum 5 (1973), 216-227. 